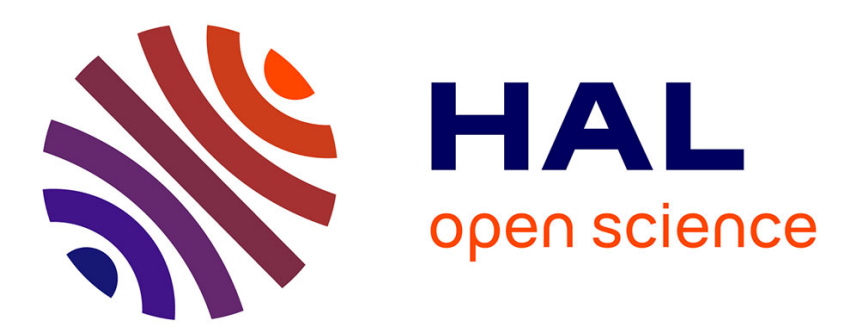

\title{
Impact of contamination on training and test error rates in statistical clustering \\ Christel Ruwet, Gentiane Haesbroeck
}

\section{To cite this version:}

Christel Ruwet, Gentiane Haesbroeck. Impact of contamination on training and test error rates in statistical clustering. Communications in Statistics - Simulation and Computation, 2011, 40 (03), pp.394-411. 10.1080/03610918.2010.542847 . hal-00666340

\section{HAL Id: hal-00666340 \\ https://hal.science/hal-00666340}

Submitted on 4 Feb 2012

HAL is a multi-disciplinary open access archive for the deposit and dissemination of scientific research documents, whether they are published or not. The documents may come from teaching and research institutions in France or abroad, or from public or private research centers.
L'archive ouverte pluridisciplinaire HAL, est destinée au dépôt et à la diffusion de documents scientifiques de niveau recherche, publiés ou non, émanant des établissements d'enseignement et de recherche français ou étrangers, des laboratoires publics ou privés. 


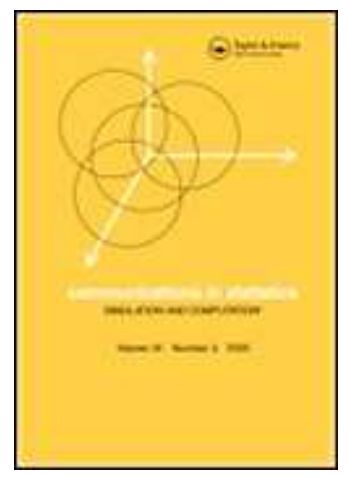

\section{Impact of contamination on training and test error rates in statistical clustering}

\begin{tabular}{|c|c|}
\hline Journal: & Communications in Statistics - Simulation and Computation \\
\hline Manuscript ID: & LSSP-2010-0117.R1 \\
\hline Manuscript Type: & Review Article \\
\hline $\begin{array}{l}\text { Date Submitted by the } \\
\text { Author: }\end{array}$ & $12-$ Oct-2010 \\
\hline Complete List of Authors: & $\begin{array}{l}\text { Ruwet, Christel; University of Liege, Mathematics } \\
\text { Haesbroeck, Gentiane; University of Liege, Mathematics }\end{array}$ \\
\hline Keywords: & $\begin{array}{l}\text { Cluster analysis, Error rate, Generalized k-means, Influence } \\
\text { function, Principal points, Robustness }\end{array}$ \\
\hline Abstract: & $\begin{array}{l}\text { The k-means algorithm is one of the most common nonhierarchical } \\
\text { clustering methods. However, this procedure is not robust with } \\
\text { respect to atypical observations. Alternative techniques have thus } \\
\text { been introduced, e.g. the generalized k-means procedure. In this } \\
\text { paper, focus is on the error rate these procedures achieve when } \\
\text { one expects the data to be distributed according to a mixture } \\
\text { distribution. Two different definitions of the error rate are under } \\
\text { consideration, depending on the data at hand. }\end{array}$ \\
\hline \multicolumn{2}{|c|}{$\begin{array}{l}\text { Note: The following files were submitted by the author for peer review, but cannot be converted } \\
\text { to PDF. You must view these files (e.g. movies) online. }\end{array}$} \\
\hline \multicolumn{2}{|c|}{ LSPP-2010-0117-RuwetHaesbroeck_Revision.tex } \\
\hline
\end{tabular}

\section{SCHOLARONE ${ }^{\text {M }}$ Manuscripts}




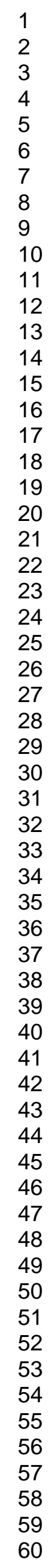
4 6

60 


\title{
Impact of contamination on training and test error rates in statistical clustering
}

C. Ruwet ${ }^{1}$ and G. Haesbroeck

University of Liège

\begin{abstract}
The $k$-means algorithm is one of the most common nonhierarchical methods of clustering. It aims to construct clusters in order to minimize the within cluster sum of squared distances. However, as most estimators defined in terms of objective functions depending on global sums of squares, the $k$-means procedure is not robust with respect to atypical observations in the data. Alternative techniques have thus been introduced in the literature, e.g. the $k$-medoids method. The $k$-means and $k$-medoids methodologies are particular cases of the generalized $k$-means procedure. In this paper, focus is on the error rate these clustering procedures achieve when one expects the data to be distributed according to a mixture distribution. Two different definitions of the error rate are under consideration, depending on the data at hand. It is shown that contamination may make one of these two error rates decrease even under optimal models. The consequence of this will be emphasized with the comparison of influence functions and breakdown points of these error rates.
\end{abstract}

Key words and phrases: Clustering analysis, Error rate, Generalized $k$-means, Influence Function, Principal points, Robustness.

\section{Introduction}

Cluster analysis is useful when one wants to group together similar objects. When using nonhierarchical procedures, the number of groups is fixed and must be chosen beforehand.

\footnotetext{
${ }^{1}$ Department of Mathematics - Grande Traverse 12, B-4000 Liège, Belgium cruwet@ulg.ac.be - tel : 04/366.94.06 - fax : 04/366.95.47
} 
This number will be set to $k$ throughout the text. Then, starting with an initial partition of the objects into $k$ groups, nonhierarchical cluster algorithms proceed iteratively in order to assign each object to the closest cluster (the closeness being assessed by a given distance between the object and the center of the cluster). The usual output of these algorithms consists of $k$ centers, each of which defines naturally a corresponding cluster as the region enveloping the points closest to that particular center. More about the basic ideas of nonhierarchical methods can be found in most textbooks on multivariate analysis (e.g. Johnson and Wichern, 2007).

As mentioned above, the result of a nonhierarchical clustering method can be given via a set of $k$ points containing the $k$ centers. The most common nonhierarchical clustering technique is probably the $k$-means algorithm which aims to find $k$ centers in order to minimize the sum of the squared Euclidean distances between the observations assigned to a cluster and the mean of this cluster. However, in this paper, following García-Escudero and Gordaliza (1999), focus is on a generalization of this algorithm: the generalized $k$-means algorithm. The main idea is to replace the quadratic penalty function by another penalty function applied to the Euclidean distances. This penalty function, $\Omega: \mathbb{R}^{+} \rightarrow \mathbb{R}^{+}$, is assumed to be continuous, nondecreasing and such that $\Omega(0)=0$ and $\Omega(x)<\lim _{y \rightarrow \infty} \Omega(y)$ for all $x \in \mathbb{R}^{+}$.

As this paper focuses on the study of influence functions, it is necessary to derive the functional forms of the estimators under consideration. As far as the $k$-means procedure is concerned, the functional form of the centers simply correspond to the principal points introduced by Flury in 1990. In the more general setting where the penalty function $\Omega$ is used, the statistical functionals characterizing the centers are given by $T_{1}(F), \ldots, T_{k}(F) \in \mathbb{R}^{p}$ which are solutions of the minimization problem

$$
\left\{T_{1}(F), \ldots, T_{k}(F)\right\}=\underset{\left\{t_{1}, \ldots, t_{k}\right\} \subset \mathbb{R}^{p}}{\operatorname{argmin}} \int \Omega\left(\inf _{1 \leq j \leq k}\left\|x-t_{j}\right\|\right) d F(x)
$$

for those distributions $F$ for which this integral exists. These statistical functionals $T_{1}(F)$, $\ldots, T_{k}(F)$ will be called generalized principal points. Taking $\Omega(x)=x^{2}$ yields the classical principal points of the distribution $F$ while $\Omega(x)=x$ gives so-called $k$-medoids principal 
points of $F$. Existence, consistency and asymptotic normality of these generalized principal points were treated by Cuesta-Albertos and Matrán (1988) and Pollard (1981 and 1982). García-Escudero and Gordaliza (1999) derived robustness properties of the generalized principal points in the particular case of univariate data to be clustered into two groups. For example, they showed that any $\Omega$ function with a bounded derivative yields a bounded influence function for the estimators $T_{1}(F)$ and $T_{2}(F)$.

Assuming that $T_{1}(F), \ldots, T_{k}(F)$ are the generalized principal points of the distribution $F$, clusters, denoted as $C_{1}(F), \ldots, C_{k}(F)$, can be constructed. The $j$ th cluster consists of the region of points closer to $T_{k}(F)$ than to any other center, the closeness being assessed by the penalty function $\Omega$. If $\omega(x)$ denotes the gradient of $\Omega(\|x\|)$ (when it exists), the first-order conditions corresponding to the minimization problem (1) are given by

$$
\int_{C_{i}(F)} \omega\left(x-T_{i}(F)\right) d F(x)=0 \quad i=1, \ldots, k
$$

showing that the generalized principal points are the $\omega$-means, in the sense of Brøns et al. (1969), of the corresponding clusters. This property will be referred to as property (P) in the sequel. For example, if $\Omega(x)=x^{2}, \omega(x)=2 x$ and the first order conditions simply imply that the principal points $T_{i}(F)$ are the means on the clusters $C_{i}(F)$ for $i=1, \ldots, k$. When the gradient of $\Omega(\|x\|)$ does not exist for a finite number of points, the integral in (2) has to be split into a sum of integrals but property (P) still holds.

When clusters are constructed, one is often interested in describing the characteristics of the observations they contain. The clustering rule corresponding to the generalized $k$ means problem (1), denoted by $R_{F}^{\mathrm{GkM}}$, associates any $x \in \mathbb{R}^{p}$ to a cluster $C_{j}(F)$ as follows:

$$
R_{F}^{\mathrm{GkM}}(x)=j \Leftrightarrow j=\underset{1 \leq i \leq k}{\operatorname{argmin}} \Omega\left(\left\|x-T_{i}(F)\right\|\right) .
$$

Clustering is often confused with classification. However, there is some difference between the two. In classification, the objects to classify are assigned to pre-defined classes, whereas in clustering the classes are also to be defined. However, when a cluster analysis is performed on a mixture distribution, it is usually referred to as a statistical cluster analysis (e.g. Fraley and Raftery, 2002, Gallegos and Ritter, 2005, Qiu and Tamhane, 
2007 or García-Escudero et al., 2008). This is the context considered in this paper. The underlying distribution $F$ will therefore be assumed to be a mixture distribution of $k$ distributions $F_{1}, \ldots, F_{k}$ with prior probabilities $\pi_{1}(F), \ldots, \pi_{k}(F)$, i.e. $F=\sum_{i=1}^{k} \pi_{i}(F) F_{i}$. Each mixture component represents a sub-population which is denoted by $G_{i}, i=1, \ldots, k$. These sets $G_{i}$ can be characterized by the values of a latent random variable, $Y$, yielding the membership, i.e. $G_{i}=\{x: Y(x)=i\}$. In this setting, one hopes to end up with clusters representing the different sub-groups. In this sense, an error rate might be defined to measure, as in classification, the performance of the clustering. Often, in practice, error rates are assessed on test data while the estimations are derived on training data, yielding the so-called test set error rate. In some applications however (e.g. Dučinskas, 1995 or Mansour and McAllester, 2002), training set error rates are computed, meaning that the same data are used for deriving the estimations and for measuring the classification performance. It is well known that in the latter case, the error rate is underestimated. The aim of the paper is to analyze the impact of contamination on this training set error rate by means of influence functions and compare the behavior of the training set error rate with that of the test set error rate.

The paper is organized as follows. Section 2 derives the statistical functionals corresponding to the test and training error rates of the generalized $k$-means classification procedure and investigates their behavior graphically. In Section 3, the first order influence function of the training error rate is derived and compared with the influence function of the test error rate computed in Ruwet and Haesbroeck (2010). Finally, Section 4 defines the breakdown point of these two error rates and uses simulations to illustrate to compare them on finite samples. 


\section{Training and test error rates}

\subsection{Statistical functionals}

Any classification rule is bound to misclassify some objects. As mentioned in the Introduction, a measure of classification performance is often defined in terms of the error rate which corresponds to the probability of misclassifying observations distributed according to a given probability measure. When both the rule and the performance are derived on the same distribution, a training error rate is obtained while a test error rate is based on two distributions, a training distribution and a test (or model) distribution.

Let us now define the statistical functionals associated to the two types of error rates. First, note that all the distributions considered in this paper are mixtures, with the implicit assumption that any observation $x$ distributed according to the $i$ th component of the mixture, $F_{i}$, belongs to an underlying sub-group $G_{i}$.

The test error rate consists of setting up the clustering rule using a training distribution, $F=\sum_{i=1}^{k} \pi_{i}(F) F_{i}$, and testing the quality of this rule on a test (or model) distribution, $F_{m}=\sum_{i=1}^{k} \pi_{i}\left(F_{m}\right) F_{m, i}$. The corresponding statistical functional can therefore be written as follows:

$$
\operatorname{ER}\left(F, F_{m}\right)=\sum_{j=1}^{k} \pi_{j}\left(F_{m}\right) \mathbb{P}_{F_{m, j}}\left[R_{F}^{\mathrm{GkM}}(X) \neq j\right] .
$$

On the other hand, for the training error rate, the same distribution is used to compute and evaluate the rule leading to the following statistical functional:

$$
\mathrm{ER}(F, F)=\sum_{j=1}^{k} \pi_{j}(F) \mathbb{P}_{F_{j}}\left[R_{F}^{\mathrm{GkM}}(X) \neq j\right] .
$$

In the sequel, the test error rate will be denoted by TER while ER will refer to the training error rate. Moreover, as ER depends only on one distribution, it will be simply denoted as $\operatorname{ER}(F)$. 


\subsection{Optimality}

A classification rule is said to be optimal if its error rate reaches the same error rate as the Bayes rule (BR) given by:

$$
R_{F}^{\mathrm{BR}}(x)=j \Leftrightarrow \pi_{j}(F) f_{j}(x)>\pi_{i}(F) f_{i}(x) \forall i \neq j
$$

where $f_{1}, \ldots, f_{k}$ are the densities (whose existence is assumed) corresponding to $F_{1} \ldots, F_{k}$.

In the context of univariate data to be clustered into two groups, assuming that the penalty function $\Omega$ is strictly increasing, the clustering rule (3) leads to the following estimated clusters:

$$
\begin{aligned}
& \left.C_{1}(F)=\left\{x \in \mathbb{R}:\left|x-T_{1}(F)\right|<\left|x-T_{2}(F)\right|\right\}=\right]-\infty, \frac{T_{1}(F)+T_{2}(F)}{2}[ \\
& \left.C_{2}(F)=\right] \frac{T_{1}(F)+T_{2}(F)}{2}, \infty[
\end{aligned}
$$

assuming, w.l.o.g., that $T_{1}(F)<T_{2}(F)$. Classification based on the generalized principal points is therefore quite straightforward. One simply needs to check where an observation lies w.r.t. the threshold $C(F)=\left(T_{1}(F)+T_{2}(F)\right) / 2$. Qiu and Tamhane (2007) have proved that the generalized 2-means procedure with $\Omega(x)=x^{2}$ (classical 2-means method) is optimal under a mixture of two homoscedastic normal distributions with equivalent weights, i.e. under the model

$$
\text { (N) } F_{N} \equiv 0.5 \mathrm{~N}\left(\mu_{1}, \sigma^{2}\right)+0.5 \mathrm{~N}\left(\mu_{2}, \sigma^{2}\right) \text { with } \mu_{1}<\mu_{2} \text {. }
$$

Model (N) will be refereed to as the optimal normal mixture. This optimality result can be extended to all generalized 2-means method as Proposition 1 below shows.

Proposition 1. Under model $(\boldsymbol{N})$, the cut-off point of any generalized 2-means procedure is the midpoint of the true means, i.e. $C\left(F_{N}\right)=\frac{\mu_{1}+\mu_{2}}{2}$, where $\mu_{1}$ and $\mu_{2}$ are the means of $F_{1}$ and $F_{2}$ respectively. This leads to the optimality of all generalized 2-means procedure under $(\boldsymbol{N})$.

The first claim is easily obtained from (2) using symmetry of (N) and the odd characteristic of $\omega$ while the second is only due to the fact that $\frac{\mu_{1}+\mu_{2}}{2}$ is the cut-off point of the Bayes rule under $(\mathbf{N})$. 
It is worth mentioning that adaptations of the $k$-means procedure have been suggested in the literature (see e.g. Symons, 1981) to reach optimality under unbalanced normal mixtures. As we do not wish to restrict the computation of the influence function to the normal setting, these adapted criteria are not used here.

\subsection{Error rate under contamination}

In ideal circumstances, the training and test distributions are identical and thus the two error rates (4) and (5) coincide. In practice however, data often contain outliers and these will affect the training distribution, while the test distribution may be assumed to remain unchanged. When contamination is present, the training distribution $F$ would be better represented as a contaminated distribution $F_{\varepsilon}=(1-\varepsilon) F+\varepsilon H$, which corresponds to a proportion $1-\varepsilon$ of data distributed according to the model while the remaining fraction, $\varepsilon$, is contaminated (i.e. comes from another distribution). When computing influence functions, the distribution $H$ of interest consists of a Dirac distribution $\Delta_{x}$ having all its mass at the point $x$, yielding the so-called point-mass contamination. This is the particular case of $F_{\varepsilon}$ which is considered from now on. In this case, the contaminated prior probabilities are given by

$$
\pi_{i}\left(F_{\varepsilon}\right)=(1-\varepsilon) \pi_{i}(F)+\varepsilon \delta_{i}(x)
$$

where $\delta_{i}(x)=\mathrm{I}\left\{x \in G_{i}\right\}$, which equals 1 if $x \in G_{i}$ and 0 otherwise. Indeed, since clean data arise with a probability $1-\varepsilon$, the probability of getting clean data from the $i$ th group is $(1-\varepsilon) \pi_{i}(F)$. A mass $\varepsilon$ is then added to the contaminated group from which the outlier comes. Also, following Croux, Filzmoser and Joossens (2008), the contaminated conditional distributions take the form

$$
F_{\varepsilon, i}=\left(1-\lambda_{i, x}(\varepsilon)\right) F_{i}+\lambda_{i, x}(\varepsilon) \Delta_{x} \text { with } \lambda_{i, x}(\varepsilon)=\frac{\varepsilon \delta_{i}(x)}{\pi_{i}\left(F_{\varepsilon}\right)}
$$

yielding the following natural decomposition of $F_{\varepsilon}: F_{\varepsilon}=\pi_{1}\left(F_{\varepsilon}\right) F_{\varepsilon, 1}+\pi_{2}\left(F_{\varepsilon}\right) F_{\varepsilon, 2}$. Under point-mass contamination, the contaminated model $F_{\varepsilon}$ remains a mixture distribution.

When working with the test error rate, even if the rule may be corrupted by being estimated on a contaminated training distribution, the error rate is evaluated on the test 
distribution assumed to be clean and with prior probabilities unaffected by the contamination (in practice, they are usually estimated assuming a prospective sampling scheme). Therefore, under contamination, the statistical functional TER, as defined in (4), becomes

$$
\operatorname{TER}\left(F_{\varepsilon}, F_{m}\right)=\sum_{j=1}^{k} \pi_{j}\left(F_{m}\right) \mathbb{P}_{F_{m}, j}\left[R_{F_{\varepsilon}}^{\mathrm{GkM}} \neq j\right],
$$

where only the rule shows a dependence on the contaminated distribution $F_{\varepsilon}$. When working with the training error rate, it is not only the rule which is corrupted but also the whole definition of the error rate, including the prior probabilities, as the following expression, corresponding to equation (5), shows :

$$
\operatorname{ER}\left(F_{\varepsilon}\right)=\sum_{j=1}^{k} \pi_{j}\left(F_{\varepsilon}\right) \mathbb{P}_{F_{\varepsilon, j}}\left[R_{F_{\varepsilon}}^{\mathrm{GkM}} \neq j\right] .
$$

As in García-Escudero and Gordaliza (1999) and Qiu and Tahmane (2007), only univariate data naturally clustered into two groups $(k=2)$ will be considered in detail. Then, using (6) and (7), explicit expressions for (10) and (11) may be obtained:

$$
\operatorname{TER}\left(F_{\varepsilon}, F_{m}\right)=\pi_{1}\left(F_{m}\right)\left\{1-F_{m, 1}\left(C\left(F_{\varepsilon}\right)\right)\right\}+\pi_{2}\left(F_{m}\right) F_{m, 2}\left(C\left(F_{\varepsilon}\right)\right)
$$

and

$$
\operatorname{ER}\left(F_{\varepsilon}\right)=\pi_{1}\left(F_{\varepsilon}\right)\left\{1-F_{\varepsilon, 1}\left(C\left(F_{\varepsilon}\right)\right)\right\}+\pi_{2}\left(F_{\varepsilon}\right) F_{\varepsilon, 2}\left(C\left(F_{\varepsilon}\right)\right)
$$

where $F_{\varepsilon, i}$ and $\pi_{i}\left(F_{\varepsilon}\right), i=1,2$, are given in (8) and (9).

Figures 1 and 2 illustrate the behavior of these error rates w.r.t. the mass of contamination or w.r.t the position of the contamination under model $(\mathbf{N})$ with $\mu_{1}=-1, \mu_{2}=1$ and $\sigma=1$. More precisely, Figure 1 represents (12) and (13) for $x \in G_{1}$ and fixed to -0.5 while Figure 2 represents these quantities for $x \in G_{1}$ and $\varepsilon$ fixed to 0.1 . In both figures, the plots on the first row correspond to the 2-means clustering method while the second row is based on the 2-medoids procedure. Also, the first columns describe the behavior of the test error rate while the second columns concern the training one. In all plots, the solid line gives the optimal ER (equal to $\Phi(-\Delta / 2)$ where $\Phi$ stands here for the standard normal cdf and $\Delta$ is the distance between the true means). 

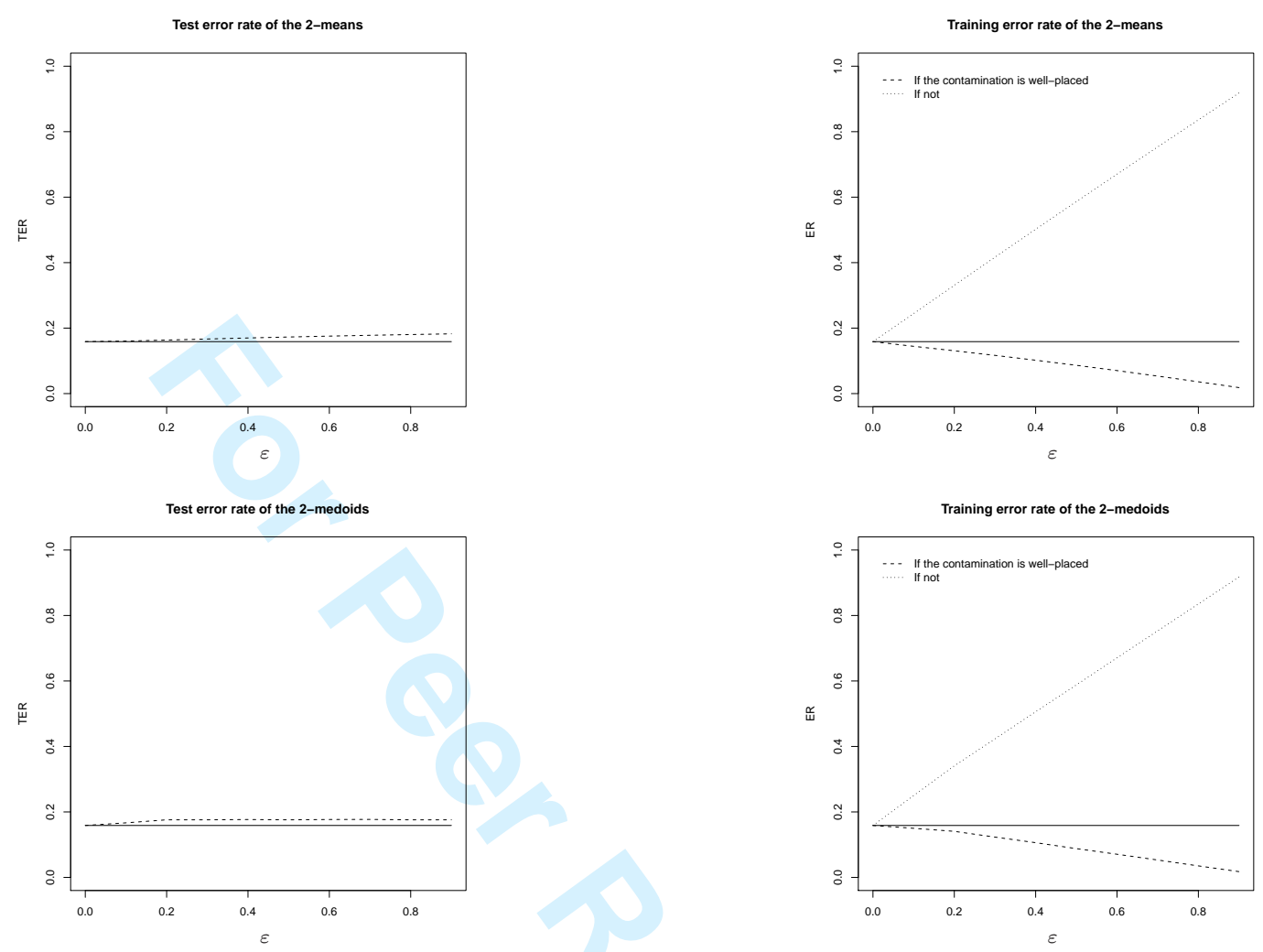

Figure 1: Optimal error rate (solid line) and contaminated error rate (dashed line) of the 2-means method (first row) and 2-medoids method (second row) when using TER (left panels) and ER (right panels) as a function of the mass of contamination $\varepsilon$ under the optimal model $(\mathbf{N})$ with $\mu_{1}=-1, \mu_{2}=1$ and $\sigma=1$. The contaminating observation is set at $x=-0.5$ and is assumed to come from $G_{1}$.

One clearly sees that contamination can only increase the error rate as soon as the test error rate is used. The behavior is quite different for the training error rate where contamination makes the error rate decrease as soon as it is well classified (this is clearly illustrated in Figure 2). Indeed, by Proposition 1, the cut-off between the two clusters is at 0 . Thus, when $x<0, x$ is in $C_{1}\left(F_{N}\right)$ and is well classified. In this case, the training error rate is smaller than the optimal error rate. As soon as $x$ becomes positive, i.e. turns out to be badly classified, the error rate increases and gets above the optimal one. When there is no contamination $(\varepsilon=0)$, the error rate of the $k$-medoids procedure attains the minimal 

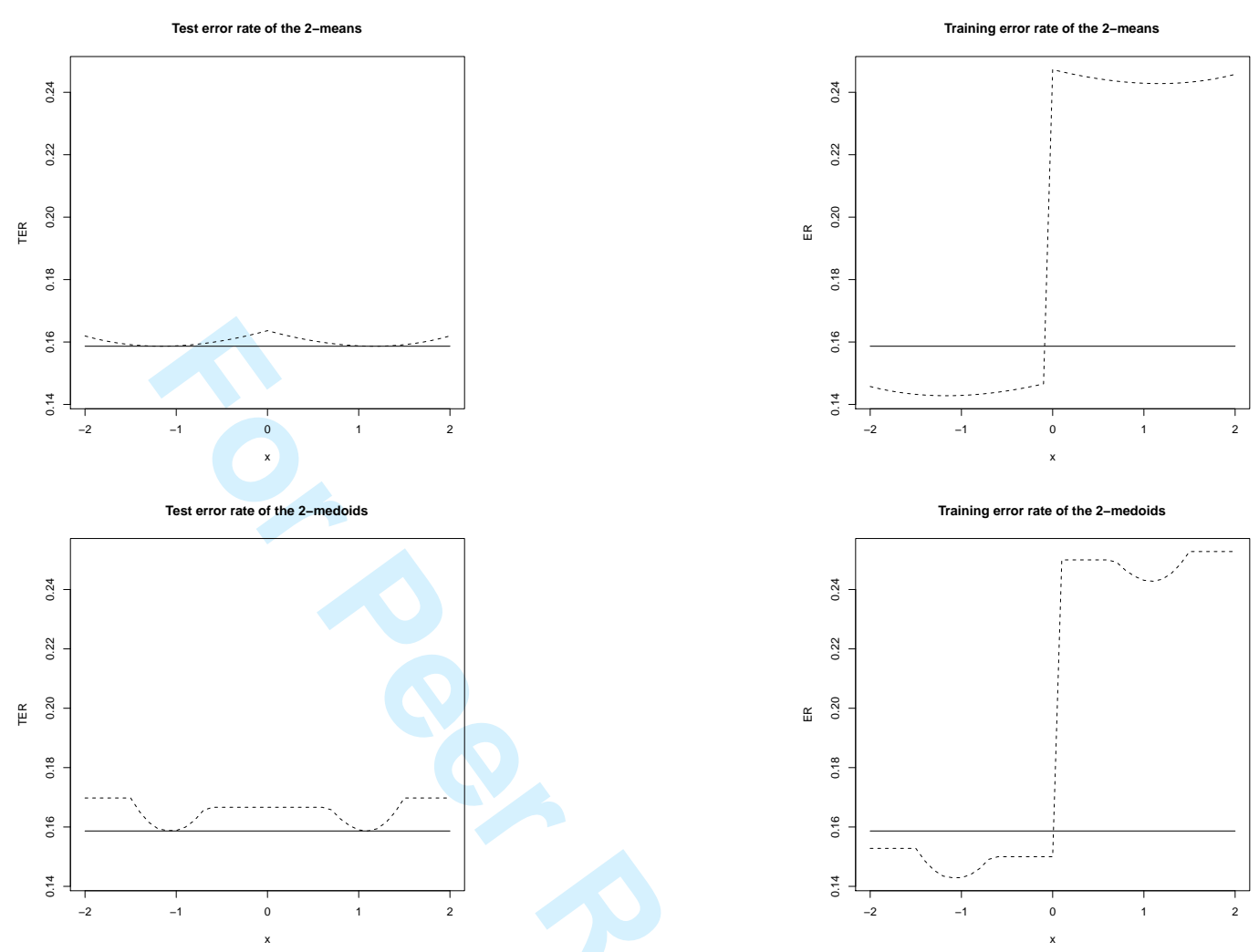

Figure 2: Optimal error rate (solid line) and contaminated error rate (dashed line) of the 2-means method (first row) and 2-medoids method (second row) when using TER (left panels) and ER (right panels) as a function of the position of the contaminating mass under model $(\mathbf{N})$ with $\mu_{1}=-1, \mu_{2}=1$ and $\sigma=1$. The percentage of contamination is set at $10 \%$ and $x \in G_{1}$.

error rate illustrating its optimality under $F_{N}$. It is also interesting to note from Figure 2 that the contaminated test error rate is the closest to the optimal error rate (without reaching it) when $x$ is closest to the generalized principal points, $T_{1}\left(F_{N}\right)$ and $T_{2}\left(F_{N}\right)$ and that the contaminated test error rate is symmetric with respect to the vertical axis $x=0$. This means that contamination that is well or badly classified has the same impact on that error rate. For the training error rate, local minima are observed in the neighborhood of $T_{1}\left(F_{N}\right)$ and $T_{2}\left(F_{N}\right)$, but symmetry does not hold anymore. In all cases, the impact of contamination is more important on the training error rate than on the test one. 
As mentioned before, property $(\mathbf{P})$ is dependent on the training distribution $F$. If $F=F_{m}$, the property holds on the test data. However, under contamination, $F=F_{\varepsilon}$, or for finite samples, $F=F_{n}$, and property $(\mathbf{P})$ does not hold for $F_{m}$. Intuitively, in cluster analysis, one would use the training error rate since only in that case the generalized principal points fully characterize the observations in the clusters. However, it has the drawback that contamination may improve the classification performance w.r.t. the one achieved under optimal models.

\section{Influence function of ER}

Roughly speaking, influence functions (Hampel et al, 1986) measure the influence that an infinitesimal contamination placed on an arbitrary point has on an estimator of interest. In Ruwet and Haesbroeck (2010), the influence function of TER is derived and is shown to vanish under the optimal model $F_{N}$. Under non optimal settings, it was observed that the influence function of TER is bounded as soon as the function $\omega$ is bounded. Moreover, as the cut-off point tends to move towards the center of the biggest group, outliers have a bigger influence when they are located in the smallest group. Also the closer the two groups are, the bigger the influence of contamination on TER is. Focus here is on the derivation of the influence function of the training error rate which is more complicated because of the presence of $\varepsilon$ everywhere in the expression (13). The influence function of the statistical functional ER at the model $F$ is defined, for those distributions for which the derivative makes sense, by

$$
\operatorname{IF}(x ; \mathrm{ER}, F)=\lim _{\varepsilon \rightarrow 0} \frac{\operatorname{ER}\left(F_{\varepsilon}\right)-\operatorname{ER}(F)}{\varepsilon}=\left.\frac{\partial}{\partial \varepsilon} \operatorname{ER}\left(F_{\varepsilon}\right)\right|_{\varepsilon=0}
$$

where $F_{\varepsilon}=(1-\varepsilon) F+\varepsilon \Delta_{x}$ and $\Delta_{x}$ is the Dirac distribution having all its mass at the point $x$.

As the statistical functional ER is a smooth function of the functionals $T_{1}$ and $T_{2}$, its influence function exists (García-Escudero and Gordaliza, 1999) and can be derived in a straightforward way. The result is given in the following proposition (the proof is given in 
the Appendix).

Proposition 2. For any mixture distribution $F$ with prior probabilities $\pi_{1}$ and $\pi_{2}$ and conditional densities $f_{1}$ and $f_{2}$, the influence function of the training error rate of the generalized 2-means method with loss-function $\Omega$ is given by

$$
\begin{array}{r}
\operatorname{IF}(x ; \mathrm{ER}, F)=\frac{1}{2}\left(\operatorname{IF}\left(x ; T_{1}, F\right)+\operatorname{IF}\left(x ; T_{2}, F\right)\right)\left\{\pi_{2}(F) f_{2}(C(F))-\pi_{1}(F) f_{1}(C(F))\right\} \\
+\operatorname{I}\{x \leq C(F)\}\left(1-2 \delta_{1}(x)\right)+\delta_{1}(x)-\operatorname{ER}(F)
\end{array}
$$

for all $x \neq C(F)$ where $C(F)=\left(T_{1}(F)+T_{2}(F)\right) / 2$ and $\delta_{1}(x)=\mathrm{I}\left\{x \in G_{1}\right\}$.

The influence function (14) depends on the influence functions of $T_{1}(F)$ and $T_{2}(F)$. These were computed for strictly increasing $\Omega$ functions by García-Escudero and Gordaliza (1999). It also depends on the belonging group and on the relative position of $x$ w.r.t. the cut-off point.

Figures 3 to 5 illustrate the behavior of the influence function (14) under some variations of the characteristics of the two sub-populations. A mixture of normal distributions (equivalent to model $(\mathbf{N})$ but with the relaxation of the constraint $\pi_{1}=\pi_{2}$ ) is selected for the representations. More specifically, $F=\pi_{1} \mathrm{~N}\left(-\mu, \sigma^{2}\right)+\pi_{2} \mathrm{~N}\left(\mu, \sigma^{2}\right)$, for given values of $\pi_{1}, \pi_{2}$ (with $\pi_{1}+\pi_{2}=1$ ), $\mu$ and $\sigma$. Of interest is the impact on the influence function of the group of the mass $x$, of the prior probabilities, of the distance between the true centers $\Delta=2 \mu$ and of the (common) dispersion within the groups, $\sigma$.

As a first general comment, one can say that, as expected, ER based on the 2-means procedure has an unbounded influence function while that of the 2-medoids is bounded. The impact of infinitesimal contamination is thus less harmful on this last procedure. However, as García-Escudero and Gordaliza (1999) show, the 2-medoids procedure can still break down when faced with a single outlier, leading to a breakdown point asymptotically equal to zero.

The influence functions show some discontinuities: one at the cut-off $C(F)$ for both $\Omega$ functions and two additional ones at $T_{1}(F)$ and $T_{2}(F)$ for the 2-medoids procedure. 

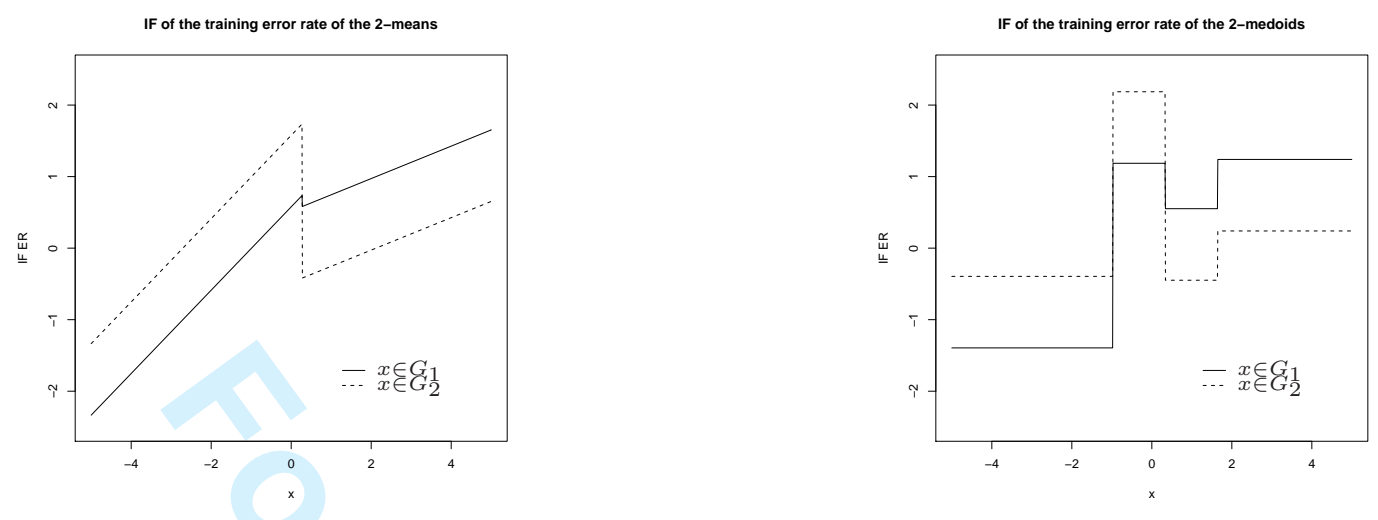

Figure 3: Influence functions of the training error rate of the 2-means (left panel) and 2medoids (right panel) methods when the model distribution is the mixture $0.2 \mathrm{~N}(-1.5,1)+$ $(1-0.2) \mathrm{N}(1.5,1)$, with a contaminating mass $x$ assumed to come from group $G_{1}$ (solid line) or group $G_{2}$ (dashed line).

These jumps come from the discontinuities already observed in the influence functions of the generalized principal points $T_{1}$ and $T_{2}$.

Now, more specifically, Figure 3 shows the effect of the group to which the contamination is assigned. One can see that a misclassified $x$, i.e. an $x$ value on the right of $C(F)$ while belonging to $G_{1}$ or an $x$ value on the left of the cut-off but belonging to $G_{2}$, results in a bigger influence than a correctly classified $x$. Indeed, the indicator functions in (14) add a value 1 to the influence function when the contamination is badly classified (the belonging group has no impact on $\operatorname{IF}\left(x ; T_{1}, F\right)$ and $\left.\operatorname{IF}\left(x ; T_{2}, F\right)\right)$. The sign of the influence is important to take into account here. Indeed, when the influence function gets negative for a given $x$, the Taylor expansion

$$
\operatorname{ER}\left(F_{\varepsilon}\right) \approx \operatorname{ER}(F)+\varepsilon \operatorname{IF}(x ; \mathrm{ER}, F), \text { for all } \varepsilon \text { small enough }
$$

implies that ER is smaller when taking the contamination into account than under the model. This really illustrates the sensitivity of classification based on the training set because contamination may improve the error rate. Indeed, under an unbalanced setting, well classified observations belonging to the smallest group (i.e. negative $x$ values in $G_{1}$ ) have a decreasing effect on the error rate. However, badly classified observations from 
the smallest group (positive $x$ values in $G_{1}$ ) increase the error rate. This behavior is also observable for the biggest group when the group masses are not too different $\left(\pi_{1} \geq 0.3\right)$. One distinguishes therefore two types of outliers: the well classified ones and the others. Well classified outliers have a positive effect on ER while badly classified ones have a negative effect on it. This is a similar phenomenon as in regression analysis where good leverage points are outliers that may improve some of the regression outputs. The positive impact of some outliers on the error rate has already been detected by Croux and Dehon (2001) in robust linear discriminant analysis.

Figure 4 illustrates how the influence function changes with respect to varying prior probabilities while assuming that $x$ belongs to the first group. First, one can see that the position of the jump corresponding to the cut-off moves towards the center of the group with the highest prior probability, as expected. Then, as far as the 2-means is concerned, one can notice that the slope of the influence function is positive for small values of $\pi_{1}$ and negative for bigger values. Another comment concerns the magnitude of the slope which is bigger (in absolute value) in the smallest group. This implies that ER based on the 2-means procedure is more sensitive to outliers in the smallest group. The 2-medoids method, on the other hand, keeps a similar behavior in all cases even if the IF gets bigger in the smallest group and smaller in the biggest group as $\pi_{1}$ increases.

It is easy to show that, in one dimension (but not in higher dimensions), the generalized principal points are affine equivariant if and only if $\omega(a x)=\rho(a) \omega(x)$ for all $x \in \mathbb{R}$, with $\rho(a) \neq 0$ for all $a \neq 0$, and this holds for the 2-means and 2-medoids principal points. The error rate is then affine invariant and it does not change when one translates both centers of the two sub-populations. However, intuitively, the error rate should decrease when the distance between the two centers gets bigger and increase otherwise. It is then interesting to visualize the effect of the variation of the distance between the two centers, $\Delta=\mu_{2}-\mu_{1}$, on the influence function. Assuming again that $x$ belongs to the first group, Figure 5 considers the impact of this distance. It shows that any well classified $x$ yields a negative influence (for both methods) while a badly classified $x$ results in positive influence. Moreover, with the 2-means approach, the effect gets bigger in absolute value as the distance gets smaller 

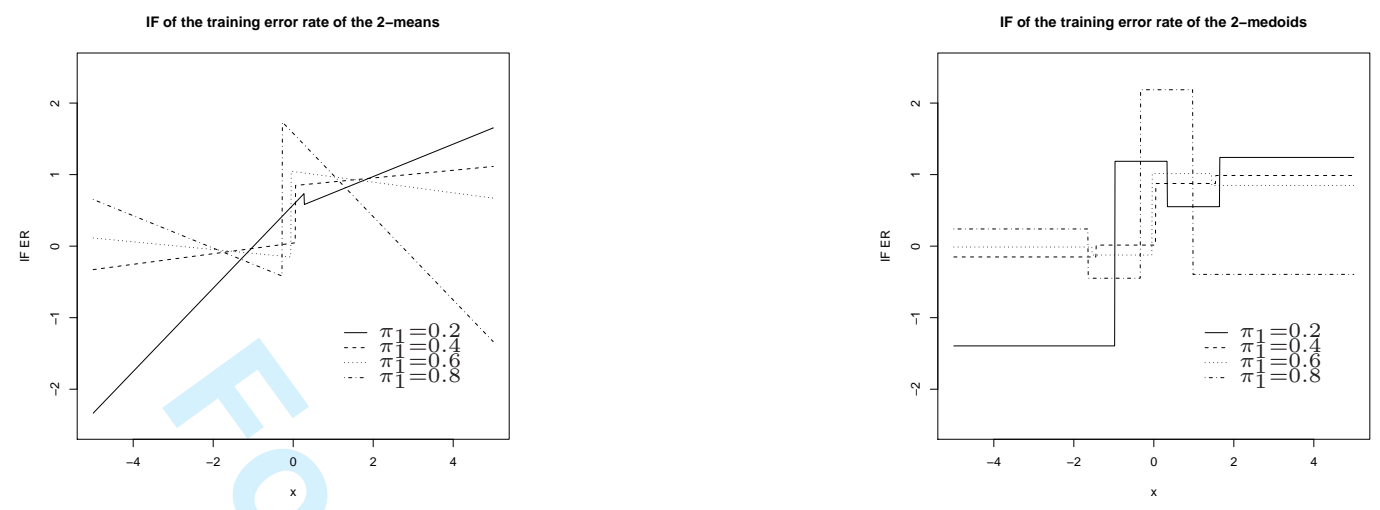

Figure 4: Influence functions of the training error rate of the 2-means (left panel) and 2medoids (right panel) methods when the model distribution is the mixture $\pi_{1} \mathrm{~N}(-1.5,1)+$ $\left(1-\pi_{1}\right) \mathrm{N}(1.5,1)$ for different values of $\pi_{1}$ when $x$ belongs to $G_{1}$.

while the 2-medoids procedure behaves differently when the observation is well or badly classified.

Similar comments hold for describing the effect of the within-group dispersion on the error rate. The corresponding figure is therefore omitted to save space.

To close the discussion on the influence function of the training error rate, it is interesting to note that the first term of (14) corresponds to the influence function of the test error rate which vanishes under optimality (Ruwet and Haesbroeck, 2010). As a consequence, the influence function of the training error rate simplifies under model $(\mathbf{N})$ as Proposition 3 shows.

Proposition 3. Under the optimal model $(\boldsymbol{N})$, the influence function of the training error rate of the generalized 2-means method reduces to

$$
\operatorname{IF}\left(x ; \mathrm{ER}, F_{N}\right)=\left\{\begin{array}{cl}
\Phi\left(\frac{\mu_{2}-\mu_{1}}{2 \sigma}\right)-\mathrm{I}\left\{x<\frac{\mu_{1}+\mu_{2}}{2}\right\} & \text { if } x \in G_{1} \\
\mathrm{I}\left\{x<\frac{\mu_{1}+\mu_{2}}{2}\right\}-\Phi\left(\frac{\mu_{1}-\mu_{2}}{2 \sigma}\right) & \text { if } x \in G_{2}
\end{array}\right.
$$

where $\Phi$ denotes here again the standard normal cumulative distribution function.

Under optimality, the influence function of ER does not vanish. This is linked to the fact that contamination can improve ER. Furthermore, it does not depend on the $\Omega$ function 

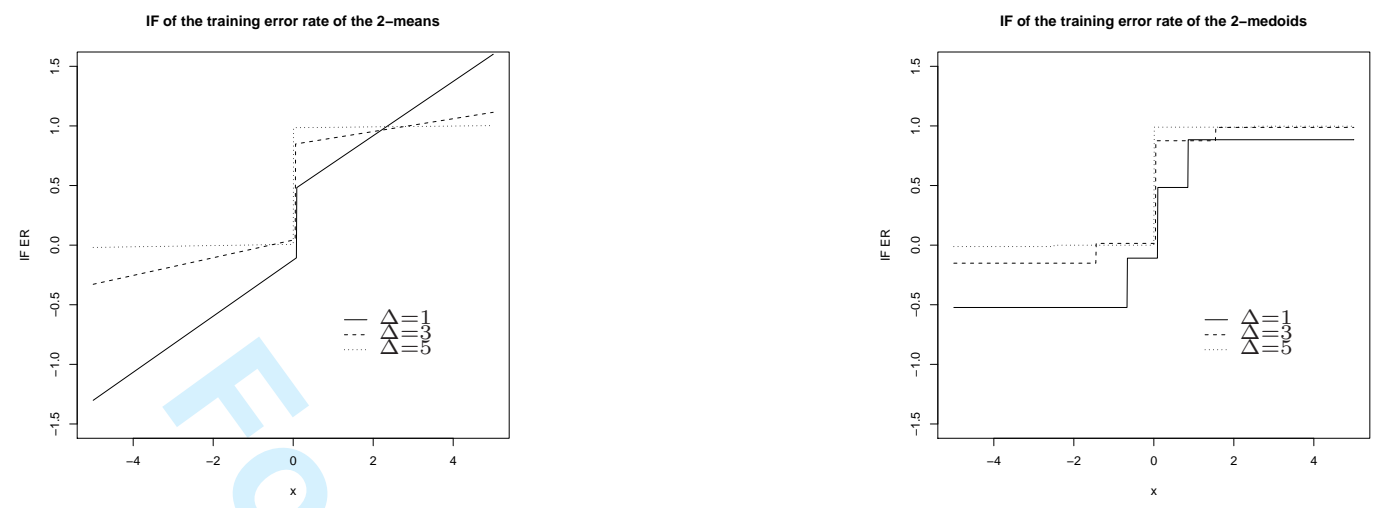

Figure 5: Influence functions of the training error rate of the 2-means (left panel) and 2-medoids (right panel) methods when the model distribution is $0.4 \mathrm{~N}(-\Delta / 2,1)+$ $0.6 \mathrm{~N}(\Delta / 2,1)$ for different values of $\Delta$ when $x$ belongs to $G_{1}$.

under consideration anymore. As $C\left(F_{N}\right)=\frac{\mu_{1}+\mu_{2}}{2}$ (see Proposition 1$)$, the influence function only depends on the distance between the two centers and on the fact that the point $x$ is well or badly classified. Indeed, one has

$$
\operatorname{IF}\left(x ; \mathrm{ER}, F_{N}\right)= \begin{cases}-\Phi\left(\frac{-\Delta}{2 \sigma}\right) & \text { if } x \text { is well classified } \\ 1-\Phi\left(\frac{-\Delta}{2 \sigma}\right) & \text { if } x \text { is badly classified }\end{cases}
$$

One can see, as before, that the influence is bigger (in absolute value) when the observation is badly classified ( since $\Delta>0$ ) while the influence is most extreme ( 0 and 1$)$ when the distance between the groups is big. In this optimal case, the influence function of ER is bounded for all choices of $\Omega$.

\section{Comparison at finite samples}

\subsection{Breakdown behavior}

Classical definitions of breakdown are based on the absolute maximal bias that an estimator can achieve under extreme contamination (e.g. Hampel, 1971 or Donoho and Huber, 1983). Intuitively, if the bias goes to infinity, the estimator is said to have broken down. However, 
when the estimator lies in a bounded space, this definition is not appropriate and an alternative one needs to be used. Several proposals exist. For example, He and Simpson (1992) say that breakdown occurs when the estimator reaches one of the bounds of its support while Genton and Lucas (2003) have argued that, in some cases, breakdown does not occur at the bounds but somewhere in the center of the parameter space. Moreover, according to them, breakdown is obtained when the set of possible values of the estimator gets restricted to a finite set while, without contamination, it could lie in an infinite set.

Breakdown in the center of the parameter space is also observed for error rates. Indeed, as soon as one observation of the training sample is sent to infinity, one of the generalized principal points goes to infinity as well, yielding a cut-off at infinity too (García-Escudero and Gordaliza, 1999). Therefore, one can surely agree that the whole procedure has broken down but the achieved error rate is not equal to 1 nor to 0 . When using TER, one entire group is badly classified while the other is well classified, leading to an error rate of either $\pi_{1}$ or $\pi_{2}$. When using ER, the corrupted observation is taken into account in measuring the classification performance. Depending on its membership, the error rate will be either $\pi_{1}-1 / n, \pi_{1}+1 / n, \pi_{2}-1 / n$ or $\pi_{2}+1 / n$. This holds for any sample of size $n$ under consideration as soon as one observation is contaminated. This means that, while error rates can theoretically take any value in $[0,1]$, under contamination, its values are restricted to a finite set of possible values. As mentioned above, this particular behavior is considered as breakdown in Genton and Lucas (2003). We may then conclude that both estimators ER and TER have a breakdown point of $1 / n$ in the sense of Genton and Lucas (2003).

This behavior is illustrated with some simulations in the next Section.

\subsection{Training set versus test set error rates}

Following Qiu and Tamhane (2007), the behavior of these error rates on finite samples will now be investigated by means of simulations. The aim is to compare the so-called test set and training set error rates with and without contamination.

In the finite sample setting, the error rates are estimated by the proportion of mis- 
classified observations (using the underlying data for the training set error rate or a test data of size $10^{5}$ for the test set error rate). Both the 2-means and 2-medoids procedures are considered, both being optimal under a balanced mixture of normal distributions. The data are generated according to the contaminated mixture

$$
F_{\varepsilon}=(1-\varepsilon)\left\{\pi_{1} N(-1,1)+\left(1-\pi_{1}\right) N(1,1)\right\}+\varepsilon \Delta_{x}
$$

with, w.l.o.g., $x \in G_{1}$. Samples of size 500 are considered with balanced $\left(\pi_{1}=0.5\right)$ and unbalanced data $\left(\pi_{1}=0.3\right.$ and $\left.\pi_{1}=0.7\right)$. The percentage of contamination is set at 0 , 0.01 and 0.05 while the position of the contamination is \pm 4 or \pm 40 . When $|x|=4$ (resp. $|x|=40$ ), one can say that there is moderate contamination (resp. extreme contamination). Moreover when $x$ is negative, it is bound to be well classified by the classification rules while $x$ positive leads to a badly classified contamination. To simplify the report of the results, the different settings under study can be summarized as follows:

- Case 0: $\varepsilon=0$ (no contamination);

- Case 1: $\varepsilon=0.01$ and $|x|=4$ (1\% of moderate contamination);

- Case 2: $\varepsilon=0.05$ and $|x|=4$ (5\% of moderate contamination);

- Case 3: $\varepsilon=0.01$ and $|x|=40$ (1\% of extreme contamination);

- Case 4: $\varepsilon=0.05$ and $|x|=40$ (5\% of extreme contamination).

Averages over 1000 simulations of the estimated training set and test set error rates are reported in Tables 1 and 2. For all error rates given in these tables, the maximal standard error is 0.045 . In Table 1 , the contamination is well classified while the reverse holds in Table 2. The optimal Bayes misclassification probability (denoted as Bayes in the tables) is also given for comparison.

Without contamination (Case 0), the 2-means procedure seems to be the best. On the other hand, the resistance to contamination of the 2-medoids method is slightly better. Indeed, this method resists to small amounts of extreme contamination (Case 3) which is 
Table 1: Simulated ER and TER of the 2-means and 2-medoids methods for balanced and unbalanced normal models with different kinds of badly classified contamination (case 0 to case 4 with negative values of $x$ ).

\begin{tabular}{|c|c|c|cc|cc|}
\hline$\pi_{1}$ & Bayes & Settings & \multicolumn{2}{|c|}{ ER } & \multicolumn{2}{c|}{ TER } \\
\hline 0.3 & \multirow{2}{*}{0.1387} & Case 0 & 0.1872 & 0.1983 & 0.1869 & 0.1985 \\
& & Case 1 & 0.1738 & 0.1938 & 0.1746 & 0.1948 \\
& & Case 2 & 0.1310 & 0.1718 & 0.1411 & 0.1780 \\
& & Case 3 & 0.2900 & 0.1946 & 0.3000 & 0.1958 \\
\hline 0.5 & 0.1587 & Case 4 & 0.2500 & 0.2500 & 0.3000 & 0.3000 \\
\hline & & Case 1 & 0.1580 & 0.1583 & 0.1616 & 0.1617 \\
& & Case 2 & 0.1595 & 0.1522 & 0.1739 & 0.1634 \\
& & Case 3 & 0.4900 & 0.1583 & 0.5000 & 0.1617 \\
\hline 0.7 & 0.1387 & Case 4 & 0.4500 & 0.4500 & 0.5000 & 0.5000 \\
\hline & Case 0 & 0.1872 & 0.1983 & 0.1869 & 0.1985 \\
& & Case 1 & 0.1935 & 0.1988 & 0.1957 & 0.2017 \\
& Case 2 & 0.2242 & 0.2023 & 0.2409 & 0.2164 \\
& Case 3 & 0.6900 & 0.1992 & 0.7000 & 0.2022 \\
& Case 4 & 0.6500 & 0.6500 & 0.7000 & 0.7000 \\
\hline
\end{tabular}


not the case for the 2-means method. Unfortunately, the 2-medoids procedure also breaks down for bigger amounts of extreme contamination. This is not surprising since Section 4.1 shows that the breakdown point is null for ER and TER. The results of the simulations show that, in practice, these estimators can resist to moderate contamination. This is not a contradiction since the breakdown point, considering the worst possible contaminated set-up, is a very pessimistic measure.

As soon as any of the two clustering procedures has broken down, the cut-off coincides with $x$. If the contamination mass is well classified (Table 1 ), $x=-4$ or $x=-40$ and all observations from $G_{1}$ bigger than $x$ are badly classified while observations from $G_{2}$ are well classified. If the error rate is computed on the training sample itself (ER), the contaminated points $(x)$ are the only observations from $G_{1}$ which are well classified. Thus the error rate corresponds to $\pi_{1}-\varepsilon$. If a non-contaminated test sample is used (TER), no observation to classify reaches $x$ (with a probability close to 1 ) and all observations coming from $G_{1}$ are badly classified, leading to an error rate of $\pi_{1}$. If the contamination mass is badly classified (Table 2), $x=4$ or $x=40$ and all observations from $G_{2}$ smaller than $x$ are badly classified while observations from $G_{1}$ are well classified. As the contamination is also badly classified, ER equals $\pi_{2}+\varepsilon$.

Under optimal model $\left(\pi_{1}=0.5\right)$, Figure 1 and Figure 2 showed that the impact of the contamination relies on the fact that it is well or badly classified. These simulations clearly show that this happens also at finite samples. When the procedure is not too affected by the contamination, one observes a smaller training set error rate under contamination than under the clean model. As expected, this is not the case for the test set error rate.

Under non-optimal models, the behavior is different if the contamination is allocated to the smallest or the biggest group. In the first case $\left(\pi_{1}=0.3\right)$, the impact of contamination is as expected: well classified outliers make the error rate decrease while badly classified ones make it increase. When $\pi_{1}=0.7$, this is not always as such. This can be explained by the fact that the cut-off is always closer to the center of the biggest group (this was illustrated on Figure 4). For example, let us consider the case of well classified contamination (Table 1). Without contamination, the cut-off would be closer to the observations from $G_{1}$ than to 
those of $G_{2}$. Adding negative observations in $G_{1}$ draws even more that cut-off towards this group. Therefore, some well classified observations from $G_{1}$ located too close to the uncontaminated cut-off may now be above the new cut-off. Finally, the non-optimality of the generalized 2-means procedure is clearly illustrated by the difference of its error rates w.r.t. the error rate of the Bayes rule.

Table 2: Simulated ER and TER of the 2-means and 2-medoids methods for balanced and unbalanced normal models with different kinds of badly classified contamination (case 0 to case 4 with positive values of $x$ ).

\begin{tabular}{|c|c|c|cc|cc|}
\hline$\pi_{1}$ & Bayes & Settings & \multicolumn{2}{|c|}{ ER } & \multicolumn{2}{c|}{ TER } \\
\hline 0.3 & \multirow{2}{*}{0.1387} & Case 0 & 0.1872 & 0.1983 & 0.1869 & 0.1985 \\
& & Case 1 & 0.2073 & 0.2137 & 0.1981 & 0.2051 \\
& & Case 2 & 0.3052 & 0.2854 & 0.2596 & 0.2397 \\
& & Case 3 & 0.7100 & 0.2156 & 0.7000 & 0.2071 \\
\hline 0.5 & \multirow{2}{*}{0.1587} & Case 4 & 0.7500 & 0.7500 & 0.7000 & 0.7000 \\
\hline & & Case 1 & 0.1595 & 0.1600 & 0.1612 & 0.1616 \\
& & Case 2 & 0.2201 & 0.2069 & 0.1757 & 0.1649 \\
& & Case 3 & 0.5100 & 0.1684 & 0.5000 & 0.1617 \\
\hline 0.7 & 0.1387 & Case 4 & 0.5500 & 0.5500 & 0.5000 & 0.5000 \\
& & Case 0 & 0.1872 & 0.1983 & 0.1869 & 0.1985 \\
& Case 1 & 0.1814 & 0.1989 & 0.1729 & 0.1914 \\
& & Case 2 & 0.1873 & 0.2089 & 0.1407 & 0.1670 \\
& Case 3 & 0.3100 & 0.1992 & 0.3000 & 0.1918 \\
& Case 4 & 0.3500 & 0.3500 & 0.3000 & 0.3000 \\
\hline
\end{tabular}




\subsection{Error rate in clustering and in discrimination}

Throughout this paper, it was assumed that the group membership was known. In practice though, other classification techniques would be more appropriate in that case, cluster analysis being mostly applied when there is no available information on the underlying groups. Nevertheless, assuming that a classification is derived from a clustering technique, it would be of interest to compare its error rate with the one achieved by a classical discriminant analysis. In this section, simulations will be carried out to compare the classification performance of the generalized $k$-means procedure and that of Fisher discriminant analysis and a robust version of it (obtained by using the robust Minimum Covariance Determinant location and scatter estimators as advocated by Croux, Filzmozer and Joossens, 2008). The chosen models for the simulations are balanced mixtures of normal distributions with standard deviation 1 (denoted as N), Student with 3 degrees of freedom (S) or log-normal with scale parameter $0.5(\mathrm{LN})$ distributions (all these models translated in order to get a center of -1 for the first group and 1 for the second one). As before, contamination (both moderate and extreme and well and badly placed) is also considered. However, Table 3 only lists the results for extreme and well classified contamination.

Let us first consider the clean setting (case 0). Under normality, the clustering procedures and Fisher analysis are equivalent. The symmetric S model leads to similar error rates of the 2-medoids procedure and the classical Fisher discriminant rule, while the 2means method results in slightly bigger error rates due to the presence of some observations in the tails. The reverse holds under the log-normal model. Under contaminated models, one can see that the classical Fisher rule, as the 2-medoids one, resists to $1 \%$ of contamination (case 3) which is not the case of the 2-means procedure. However, only the robust version of the Fisher rule gets reasonable error rates under $5 \%$ of extreme contamination (case 4$)$. 
Table 3: Simulated ER and TER of the 2-means and 2-medoids methods as well as the classical and robust Fisher discriminant rules for balanced models (normal, student and log-normal) with different kinds of well classified contamination (negative values of $x$ ).

\begin{tabular}{|c|c|c|cc|cc|cc|}
\hline \multirow{2}{*}{ Models } & \multirow{2}{*}{$\begin{array}{c}\text { ER of } \\
\text { Bayes }\end{array}$} & Settings & \multicolumn{2}{|c|}{ ER } & \multicolumn{2}{c|}{ TER } & \multicolumn{2}{c|}{ Fisher } \\
& & 2-means & 2-med & 2-means & 2-med & classical & robust \\
\hline \multirow{2}{*}{$\mathrm{N}$} & \multirow{2}{*}{0.1587} & Case 0 & 0.1595 & 0.1600 & 0.1612 & 0.1616 & 0.1607 & 0.1612 \\
& & Case 3 & 0.4900 & 0.1583 & 0.5000 & 0.1617 & 0.1781 & 0.1612 \\
& & Case 4 & 0.4500 & 0.4500 & 0.5000 & 0.5000 & 0.4149 & 0.1612 \\
\hline \multirow{2}{*}{$\mathrm{S}$} & \multirow{2}{*}{0.1955} & Case 0 & 0.2082 & 0.1963 & 0.2095 & 0.1981 & 0.1977 & 0.1976 \\
& & Case 3 & 0.4900 & 0.1945 & 0.5000 & 0.1982 & 0.1977 & 0.1976 \\
& & Case 4 & 0.4500 & 0.4500 & 0.5000 & 0.5000 & 0.4140 & 0.1976 \\
\hline \multirow{2}{*}{ LN } & \multirow{2}{*}{0.0254} & Case 0 & 0.0377 & 0.0453 & 0.0385 & 0.0459 & 0.0334 & 0.0526 \\
& & Case 3 & 0.4900 & 0.0450 & 0.5000 & 0.0464 & 0.0688 & 0.0540 \\
& & Case 4 & 0.4500 & 0.4500 & 0.5000 & 0.5000 & 0.5000 & 0.0570 \\
\hline
\end{tabular}




\section{Conclusion}

This paper studied the error rate as a measure of performance of a classification rule resulting from the generalized $k$-means algorithm, which is a generalization of the classical $k$-means procedure. Two definitions of the error rate were considered, depending on the knowledge or not of a reference distribution, the training and test error rates.

Their influence functions have been computed under general mixture distributions in order to measure their robustness w.r.t. small amounts of contamination. It was shown that contamination may make the training error rate decrease even under optimal models (balanced mixtures of normal distributions). This implies that the influence function of the training error rate does not vanish under optimality while that is the case for the test error rate (as expected under optimal models). The study of the influence function of the training error rate showed that observations that are badly classified have a bigger influence than well classified ones. Moreover, this influence is more important in the smallest group and/or when the groups are not too far away from each other. However, the main conclusion is that any penalty function with a bounded derivative leads to a bounded influence function of the training error rate.

Boundedness of the influence function is one robustness characteristic but a more global measure of robustness is given by the breakdown point. Following the definition of Genton and Lucas (2003), ER and TER have been shown to have a breakdown point asymptotically equal to zero. Simulations were used to illustrate the robustness of ER and TER on finite samples and to compare the generalized $k$-means clustering method with Fisher's linear discriminant analysis. The latter was found to yield error rates which are quite comparable to the ones attained with the 2-medoids method. Only the robust version of the Fisher's linear discriminant rule resists to $5 \%$ percent of extreme oultiers. To improve the resistance of the generalized $k$-means procedure to contamination, Cuesta-Albertos, Gordaliza and Matrán (1997) introduced the trimmed $k$-means procedure. 


\section{Appendix}

\section{Proof of Proposition 2} ward:

By definition, $\operatorname{IF}(x ; \mathrm{ER}, F)=\left.\frac{\partial}{\partial \varepsilon} \operatorname{ER}\left(F_{\varepsilon}\right)\right|_{\varepsilon=0}$. The following derivatives are straightfor-

$$
\left.\frac{\partial}{\partial \varepsilon} \pi_{i}\left(F_{\varepsilon}\right)\right|_{\varepsilon=0}=\delta_{i}(x)-\pi_{i}(F) \text { and }\left.\frac{\partial}{\partial \varepsilon} \lambda_{i, x}(\varepsilon)\right|_{\varepsilon=0}=\frac{\delta_{i}(x)}{\pi_{i}(F)} .
$$

This leads to the derivative of $F_{\varepsilon, i}\left(C\left(F_{\varepsilon}\right)\right)$ :

$$
\frac{\delta_{i}(x)}{\pi_{i}(F)}\left\{\Delta_{x}(C(F))-F_{i}(C(F))\right\}+f_{i}(C(F)) \frac{1}{2}\left\{\operatorname{IF}\left(x ; T_{1}, F\right)+\operatorname{IF}\left(x ; T_{2}, F\right)\right\} .
$$

As

$$
\begin{aligned}
\left.\frac{\partial}{\partial \varepsilon} \operatorname{ER}\left(F_{\varepsilon}\right)\right|_{\varepsilon=0}=\left.\frac{\partial}{\partial \varepsilon} \pi_{1}\left(F_{\varepsilon}\right)\right|_{\varepsilon=0} & \left\{1-F_{1}(C(F))\right\}-\left.\pi_{1}(F) \frac{\partial}{\partial \varepsilon} F_{\varepsilon, 1}\left(C\left(F_{\varepsilon}\right)\right)\right|_{\varepsilon=0} \\
& +\left.\frac{\partial}{\partial \varepsilon} \pi_{2}\left(F_{\varepsilon}\right)\right|_{\varepsilon=0} F_{2}(C(F))+\left.\pi_{2}(F) \frac{\partial}{\partial \varepsilon} F_{\varepsilon, 2}\left(C\left(F_{\varepsilon}\right)\right)\right|_{\varepsilon=0},
\end{aligned}
$$

one gets the influence function by plugging the derivatives of each component in this expression.

\section{References}

Brøns HK, Brunk HD, Franck WE and Hanson DL (1969) Generalized means and associated families of distributions. The Annals of Mathematical Statistics 40:339-355.

Croux C and Dehon C (2001) Robust linear discriminant analysis using S-estimators. The Canadian Journal of Statistics 29:473-492.

Croux C, Filzmoser P and Joossens K (2008) Classification efficiencies for robust linear discriminant analysis. Statistica Sinica 18:581-599.

Cuesta-Albertos JA, Gordaliza A and Matrán C (1997) Trimmed k-means: an attempt to robustify quantizers. The Annals of Statistics 25:553-576.

Cuesta-Albertos JA and Matrán C (1988) The strong law of large numbers for k-means and best possible nets of Banach valued random variables. Probability theory and related fields 78:523-534. 
Donoho DL and Huber PJ (1983) The notion of breakdown point. In A Festschrift for Erich L. Lehmann (eds PJ Bickel, KA Doksum ajd JL Hodges, Jr), 157-184. Belmont: Wadsworth.

Dučinskas K (1995) Optimal training sample allocation and asymptotic expansions for error rates in discriminant analysis. Acta Applicandae Mathematicae 38:3-11.

Flury BA (1990) Principal points. Biometrika 77:33-41.

Fraley C and Raftery AE (2002) Model-based clustering, discriminant analysis, and density estimation. Journal of the American Statistical Association 97:611-631.

Gallegos MT and Ritter G (2005) A robust method for cluster analysis. The Annals of Statistics 33:347-380.

García-Escudero LA and Gordaliza A (1999) Robustness properties of $k$ means and trimmed $k$ means. Journal of the American Statistical Association 94:956-969.

García-Escudero LA, Gordaliza A, Matrán C and Mayo-Iscar A (2008) A general trimming approach to robust cluster analysis. The Annals of Statistics 36:1324-1345.

Geton MG and Lucas A (2003) Comprehensive definitions of breakdown points for independent and dependent observations. Journal of the Royal Statistical Society. Ser B 65:81-94.

Hampel FR (1971) A general qualitative definition of robustness. The Annals of Statistics 42:1887-1896.

Hampel FR, Ronchetti EM, Rousseeuw PJ and Stahel WA (1986) Robust statistics: The Approach Based on Influence Functions John Wiley and Sons, New-York

He X and Simpson DG (1992) Robust direction estimation. The Annals of Statistics 20:351-369.

Johnson RA and Wichern DW (2007) Applied multivariate statistical analysis $6^{\text {th }}$. Pearson Prentice Hall, Upper Saddle River.

Mansour Y and McAllester D (2002) Boosting using branching programs. Journal of computer and system sciences 64:103-112. 
Pison G, Van Aelst S. (2004). Diagnostic plots for robust multivariate methods. Journal of Computational and Graphical Statistics 13:310-329.

Pollard D (1981) Strong consistency of $k$-means clustering. The Annals of Statistics 9:135140.

Pollard D (1982) A central limit theorem for $k$-means clustering. The Annals of Probability 10:919-926.

Qiu D and Tamhane AC (2007) A comparative study of the $k$-means algorithm and the normal mixture model for clustering: Univariate case. Journal of Statistical Planning and Inference 137:3722-3740.

Ruwet C and Haesbroeck G (2010) Classification performance of $k$-means. Preprint of the University of Liège.

Symons MJ (1981) Clustering criteria and multivariate normal mixtures. Biometrics 37:3543 


\title{
LSPP-2010-0117: Impact of contamination on training and test error rates in statistical clustering
}

\author{
Reply to reviewer 1
}

First of all, we wish to thank you for your comments and helpful suggestions. See below the responses to your specific comments.

Comment 1: You are right. We have rephrased this (page 1, two last lines).

Comment 2: This sentence has been modified as you suggested (page 2, line 15).

Comment 3: You are right again. Model-based clustering is indeed a way to perform clustering based on a fitted mixture while we really wanted to refer to the general framework of cluster analysis based on mixtures. The reference to "model-based clustering" has been suppressed.

Comment 4: Your suggestion has been taken into account and a definition of the sets $G_{i}$ is now incorporated in the definition of the mixture distribution under consideration (page 4, line 4).

Comment 5: Section 2.3 was originally written for a general contaminated distribution $F_{\varepsilon}$. However, for the computation of influence functions, only the particular case of point mass contamination needs to be considered. As we found it quite difficult to explicitly define $F_{\varepsilon, j}$ and $\pi_{i}\left(F_{\varepsilon}\right)$ in the general contamination context, we decided to focus directly on the point mass contaminated distribution. Therefore, Section 2.3 has been reorganized and the detailed treatment of $F_{\varepsilon, j}$ and $\pi_{i}\left(F_{\varepsilon}\right)$ in case of pointwise contamination appears now in the beginning of the Section. We hope that this will help the reader to better comprehend the type of contamination we are dealing with. Now, concerning the choice of $\varepsilon$-contamination we made, it corresponds to the $\varepsilon$-contamination advocated by Croux, Filzmozer and Joossens (2008) who also dealt with contamination in mixture distributions. Therefore, we thought that it was quite adequate in our setting.

Comment 6: Under contamination, the test set error rate does not attain the optimal error rate. We mention this explicitly now in the revised paper (page 10, line -7 ).

Comment 7: The word "procedure" referred to the classification based on the training set. This is more explicitly mentioned in the text (page 13, last lines).

Comment 8: True. We have added a comment to specify this restriction (page 14, line -11). 
Comment 9: Of course, we may provide the "deleted" figures (they are displayed below for your information) but we are not sure whether it is worth preparing a technical report for that. Maybe these additional figures could be simply inserted in the main text if you think that this is relevant?

Comment 10: The breakdown point defined by Genton and Lucas (2003) seems to be the appropriate tool for computing the breakdown point of a classification error rate. Indeed, one cannot observe an asymptotic bias going to infinity in this setting but breakdown can still be characterized by the fact that the error rate reaches some specific values. We added a sub-section to introduce the idea of breakdown as defined by Genton and Lucas (2003). The breakdown results of García-Escudero and Gordaliza (1999) may then be quoted to argue that a single outlier is sufficient to totally perturb the generalized principal points, and, as a direct consequence, the error rates based on them. We conclude that sub-section by mentioning that ER and TER have a breakdown point of $1 / n$, since, as soon as one worst-case outlier is introduced in the data, their possible values lie into a finite set. 

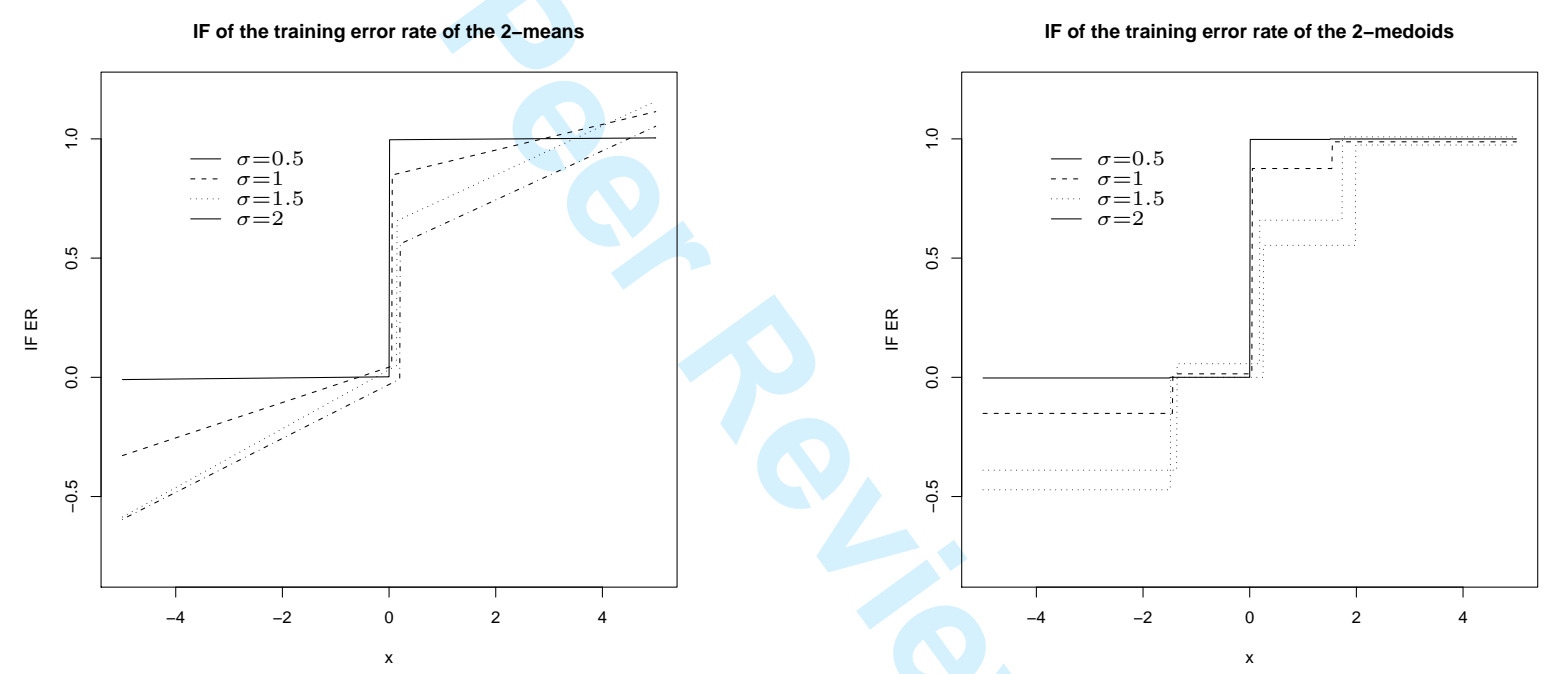

Figure 1: Influence functions of the training error rate of the 2-means (left panel) and 2-medoids (right panel) methods when the model distribution is $0.4 \mathrm{~N}\left(-1.5, \sigma^{2}\right)+$ $0.6 \mathrm{~N}\left(1.5, \sigma^{2}\right)$ for different values of $\sigma$ when $x$ belongs to $G_{1}$. 


\title{
LSPP-2010-0117: Impact of contamination on training and test error rates in statistical clustering
}

\author{
Reply to reviewer 2
}

First of all, we wish to thank you for your comments and helpful suggestions. See below the responses to your specific comments.

Comment 1: Your suggestion has been taken into account and the reference has been added (page 4, line 1).

Comment 2: That is indeed true. Until now, we had been focusing simply on the 2-means and 2medoids procedures but your question made us consider the optimality issue in more general terms. Due to the odd property of $\omega$, one can show that $T_{1}\left(F_{N}\right)+T_{2}\left(F_{N}\right)=$ $\mu_{1}+\mu_{2}$ for all generalized 2-means procedure applied to the optimal normal model. Moreover, in parallel, we could adapt Proposition 1 which now proves that optimality results directly from such an equality. To better organize the results related to the optimality issue, Proposition 1 has been transferred to Section 2.2 (while it was in Section 2.3 in the previous version). Sections 2.2 and 2.3 have been modified to take this particular re-organization into account (see in particular page 6).

Comment 3: A comment concerning the null asymptotic breakdown point of 2-medoids procedure has been added in Section 3 (page 12, lines -5).

Comment 5: We have specified the values of the parameters (page 22, line 11).

Typos that you detected have been corrected. Moreover, another thorough spell check and a careful reading of the revised paper have allowed to find other typos and to improve, we hope, the text. 
Training error rate of the 2-means

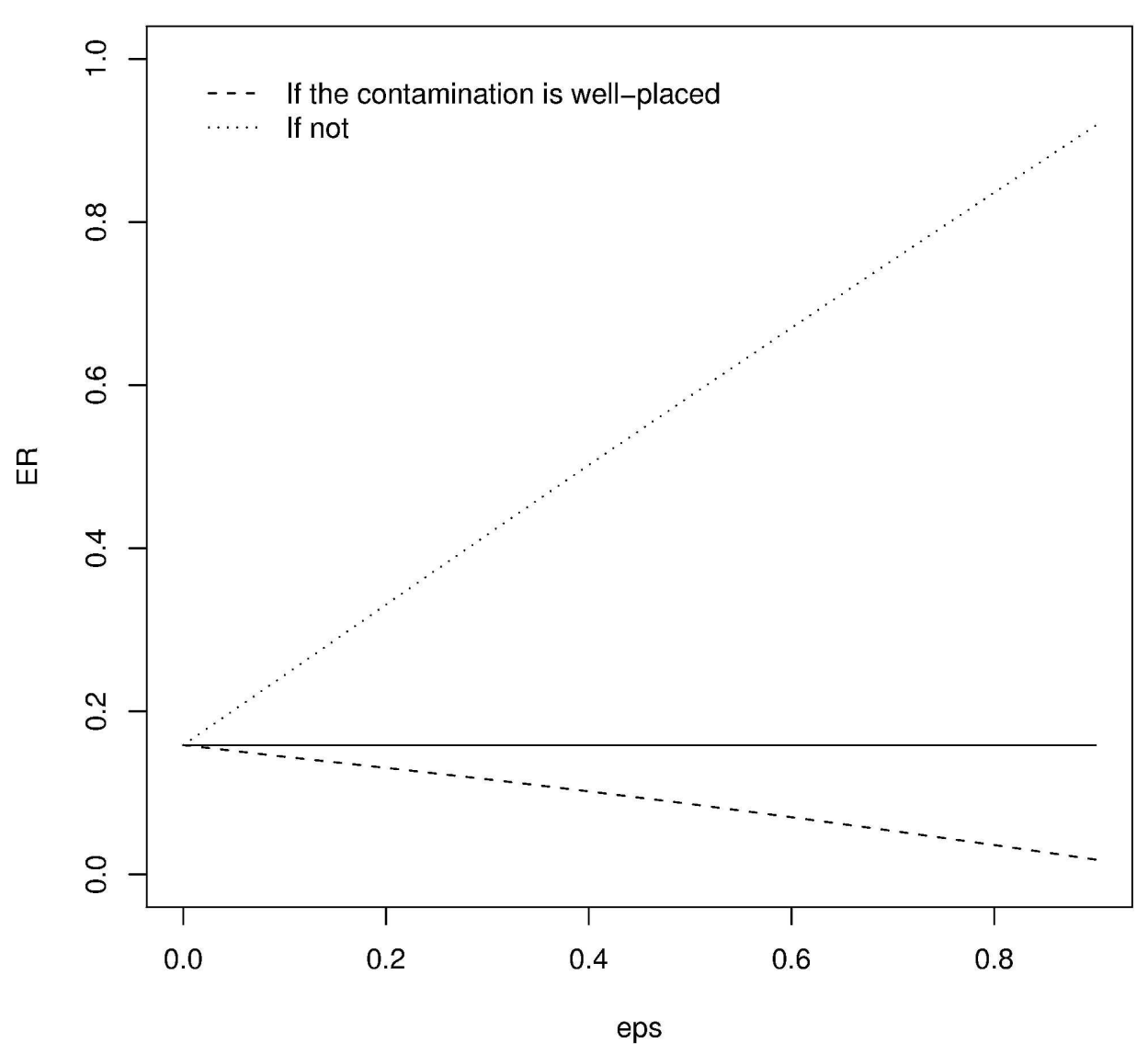

$177 \times 177 \mathrm{~mm}(600 \times 600 \mathrm{DPI})$ 
Training error rate of the 2-medoids

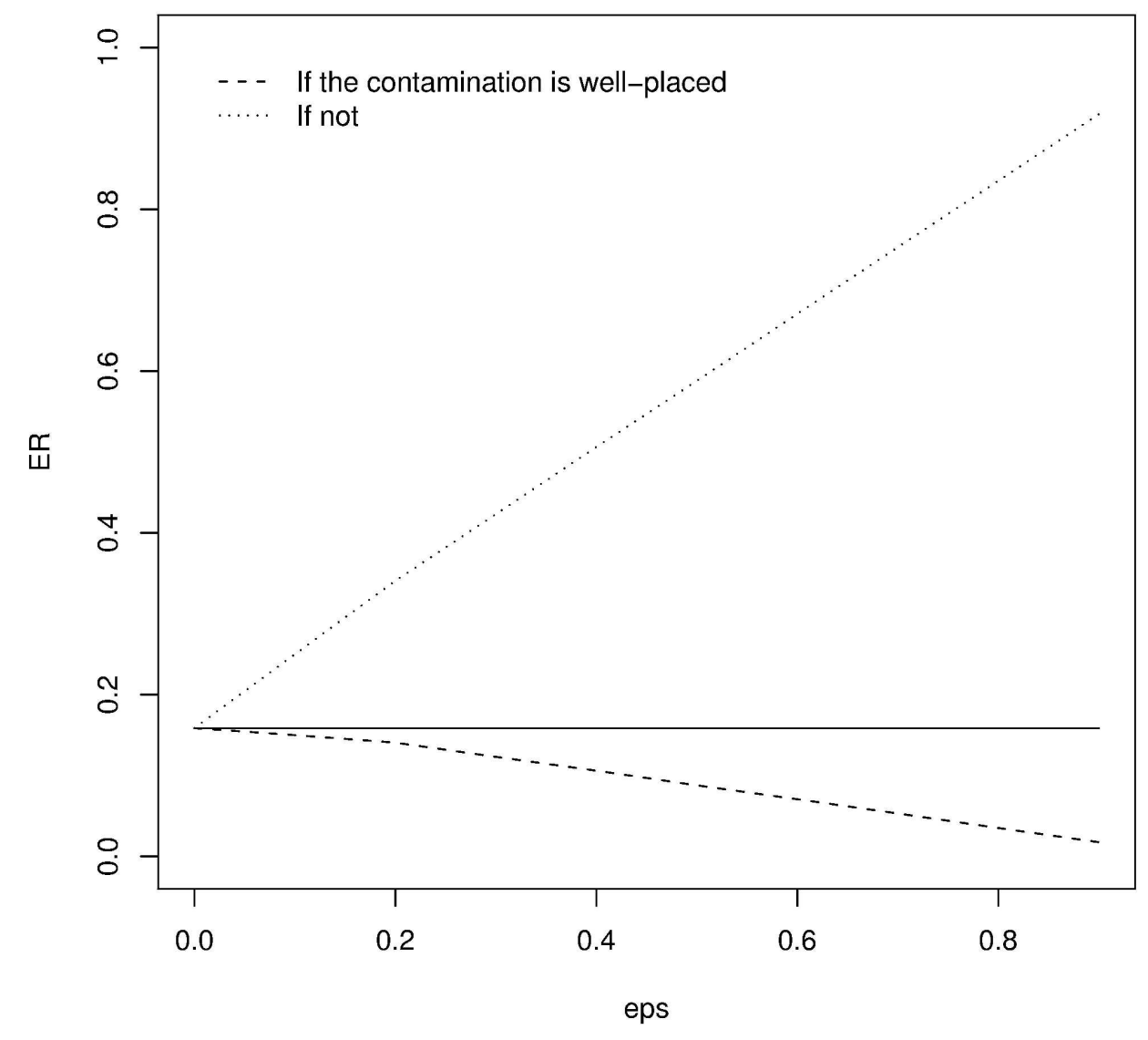

$177 \times 177 \mathrm{~mm}(600 \times 600 \mathrm{DPI})$ 
1

2

3

4

5

6

7

8

10

11

12

14

15

16

17

18

19

20

21

22

23

24

25

26

27

28

29

30

31

32

33

34

35

36

37

38

39

40

41

42

43

44

45

46

47

48

49

50

51

52

53

54

55

56

57

58

59

60
Test error rate of the 2-means

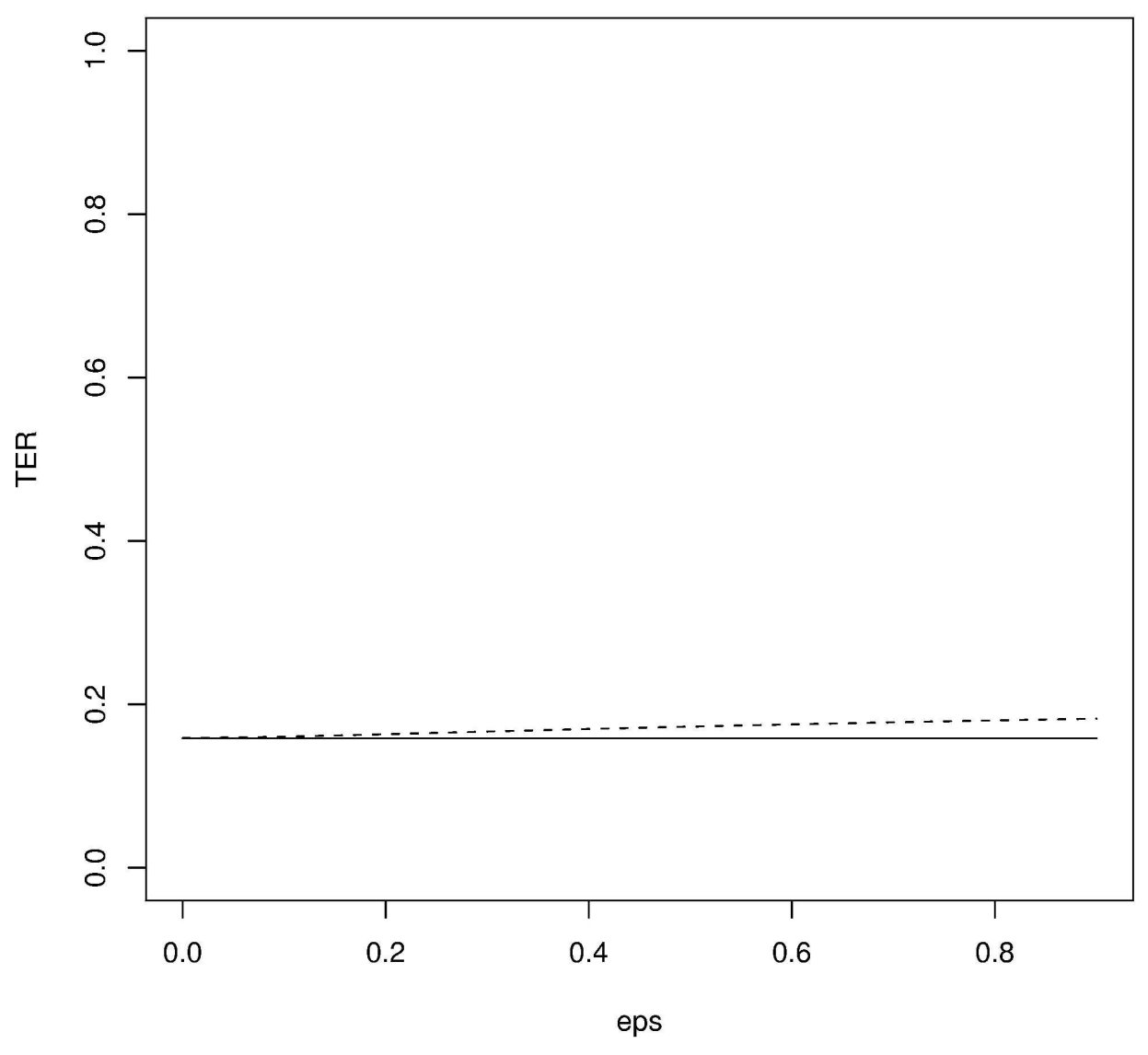

$177 \times 177 \mathrm{~mm}(600 \times 600 \mathrm{DPI})$ 
Test error rate of the 2-medoids

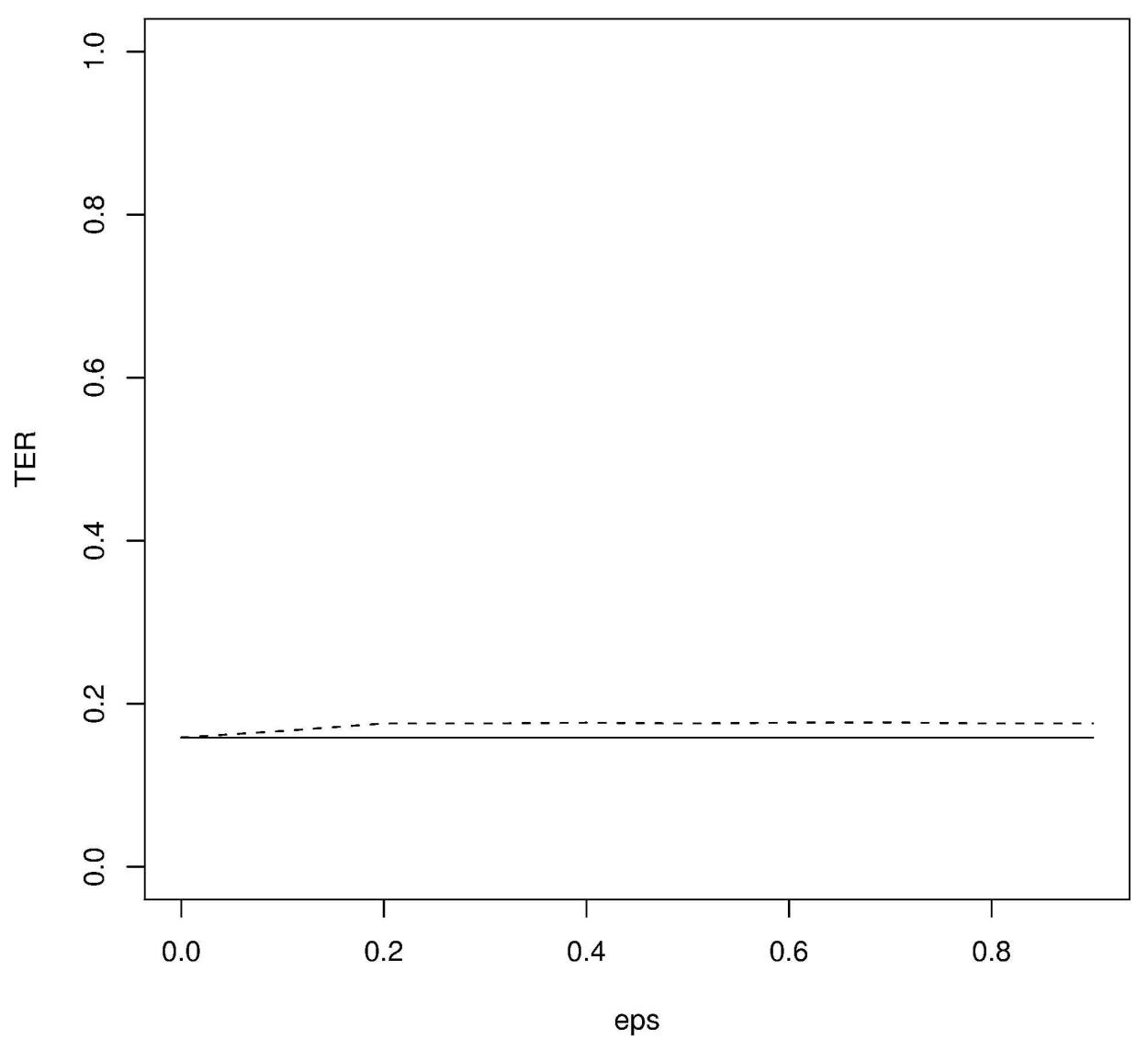

$177 \times 177 \mathrm{~mm}(600 \times 600 \mathrm{DPI})$ 
1

2

3

4

5

6

7

8

10

11

12

14

15

16

17

18

19

20

21

22

23

24

25

26

27

28

29

30

31

32

33

34

35

36

37

38

39

40

41

42

43

44

45

46

47

48

49

50

51

52

53

54

55

56

57

58

59

60

Training error rate of the 2-means

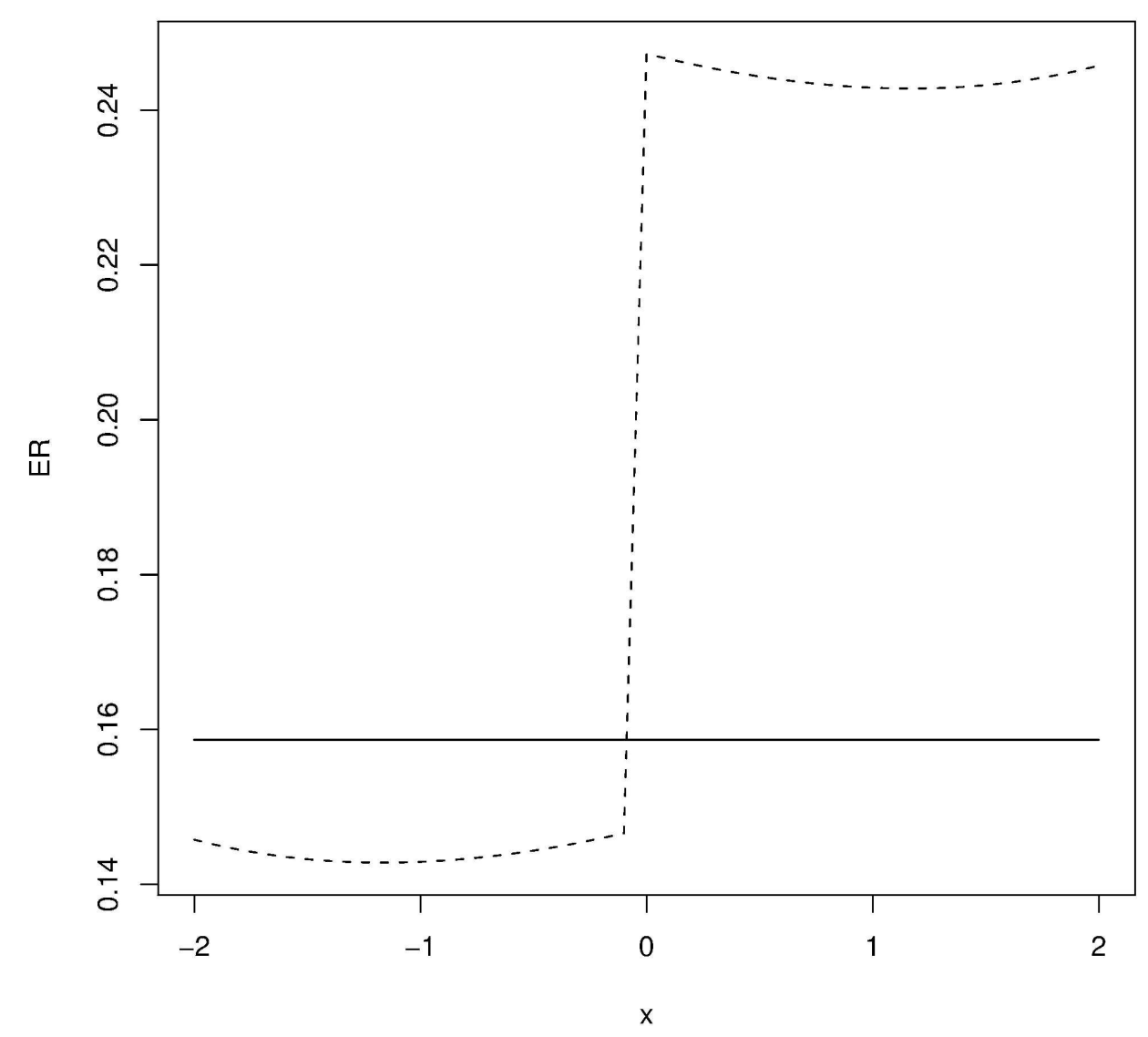

$177 \times 177 \mathrm{~mm}(600 \times 600 \mathrm{DPI})$ 
Training error rate of the 2-medoids

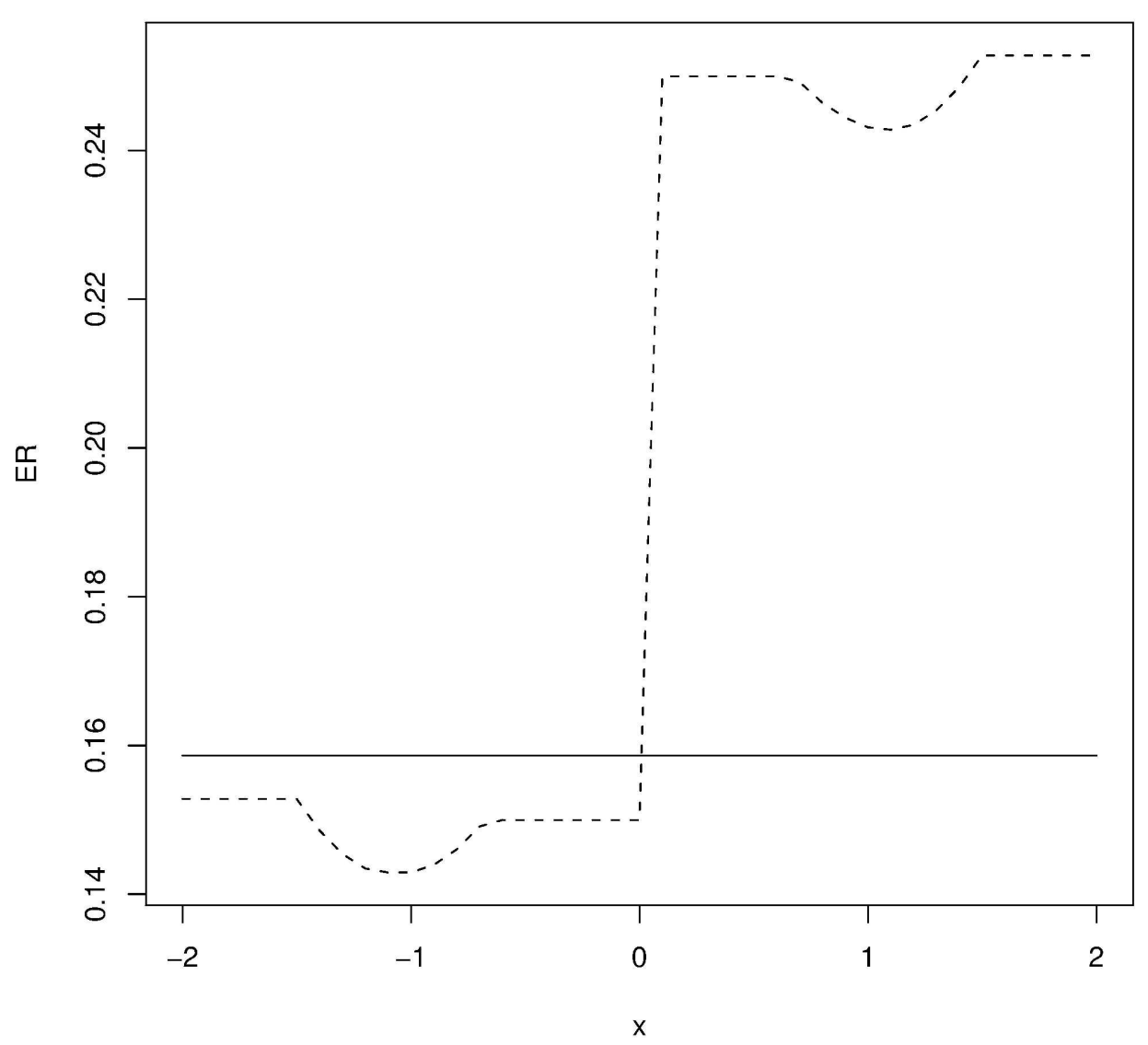

$177 \times 177 \mathrm{~mm}(600 \times 600 \mathrm{DPI})$ 
1

2

3

4

5

6

7

8

10

11

12

14

15

16

17

18

19

20

21

22

23

24

25

26

27

28

29

30

31

32

33

34

35

36

37

38

39

40

41

42

43

44

45

46

47

48

49

50

51

52

53

54

55

56

57

58

59

60
Test error rate of the 2-means

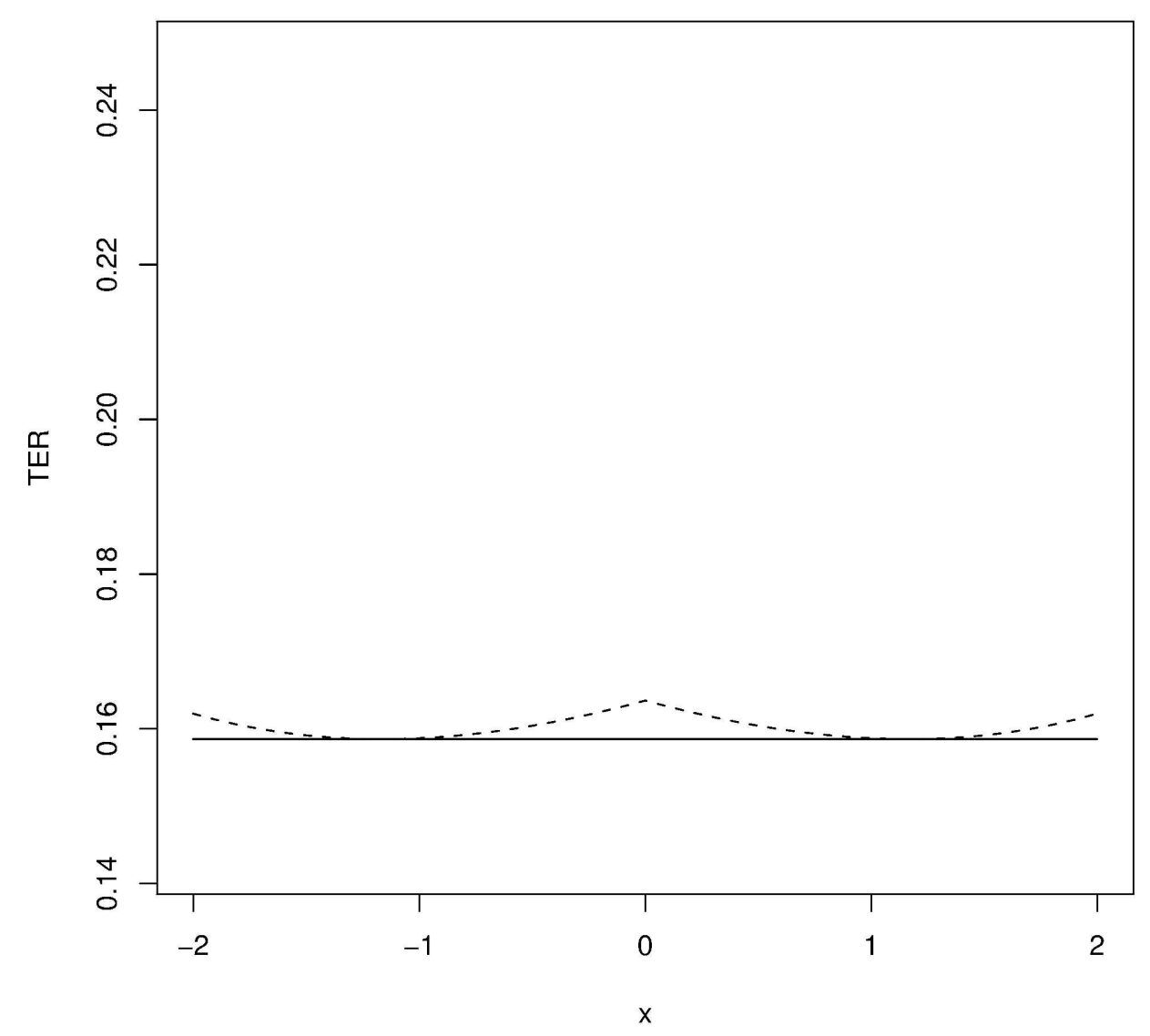

$177 \times 177 \mathrm{~mm}(600 \times 600 \mathrm{DPI})$ 
Test error rate of the 2-medoids

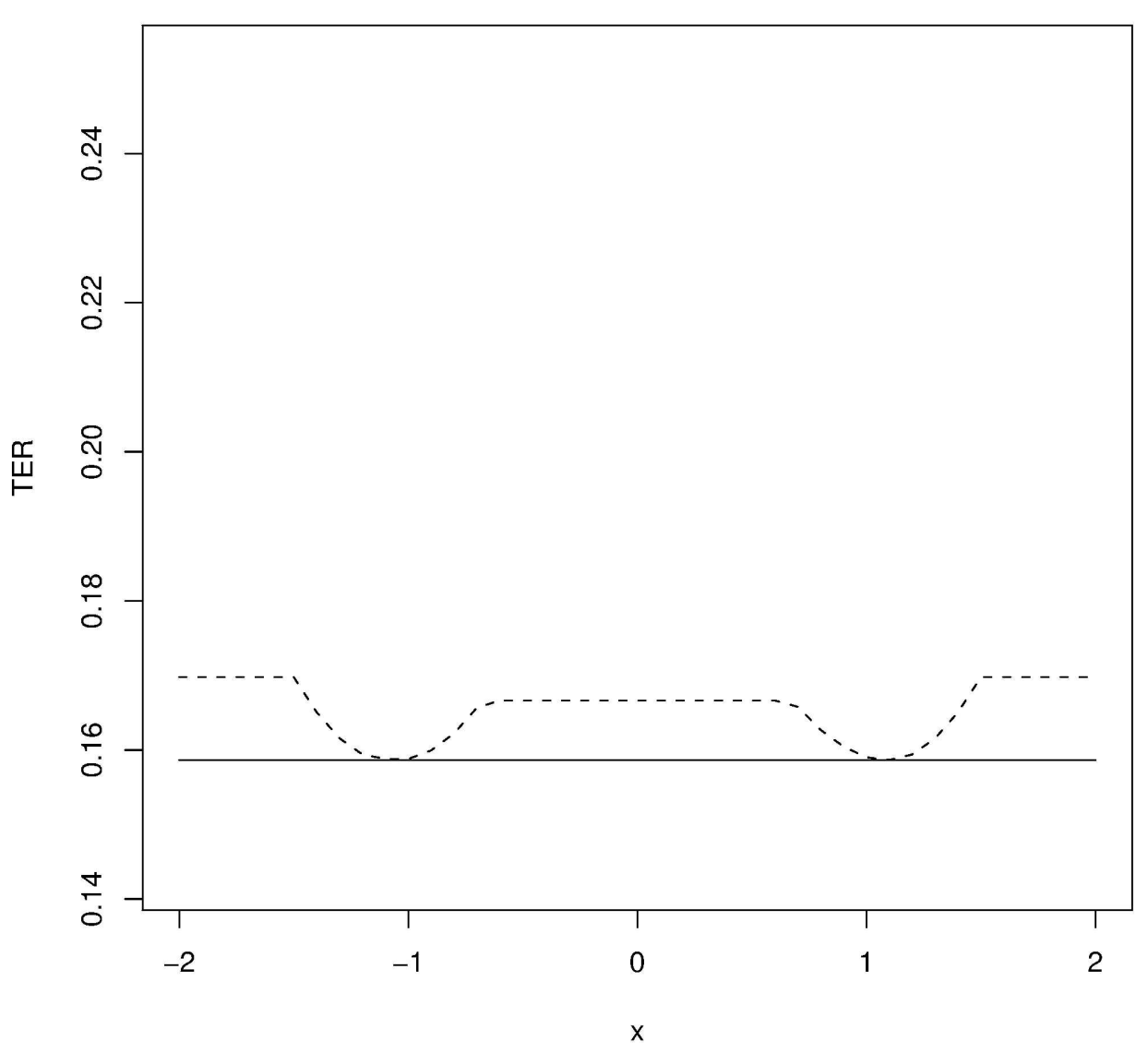

$177 \times 177 m m(600 \times 600$ DPI $)$ 
IF of the training error rate of the 2-means

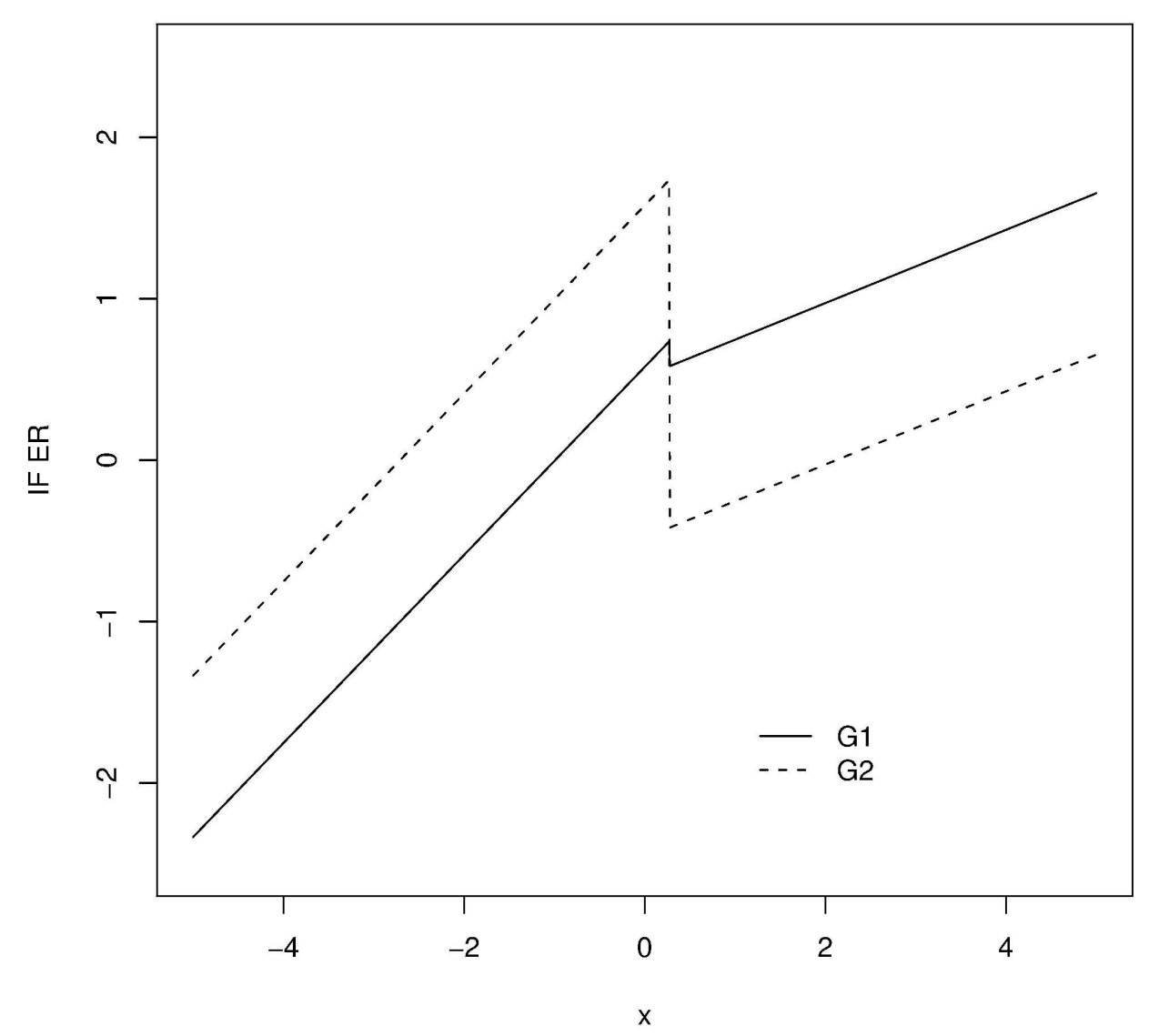

$177 \times 177 m m(600 \times 600$ DPI $)$ 
IF of the training error rate of the 2-medoids

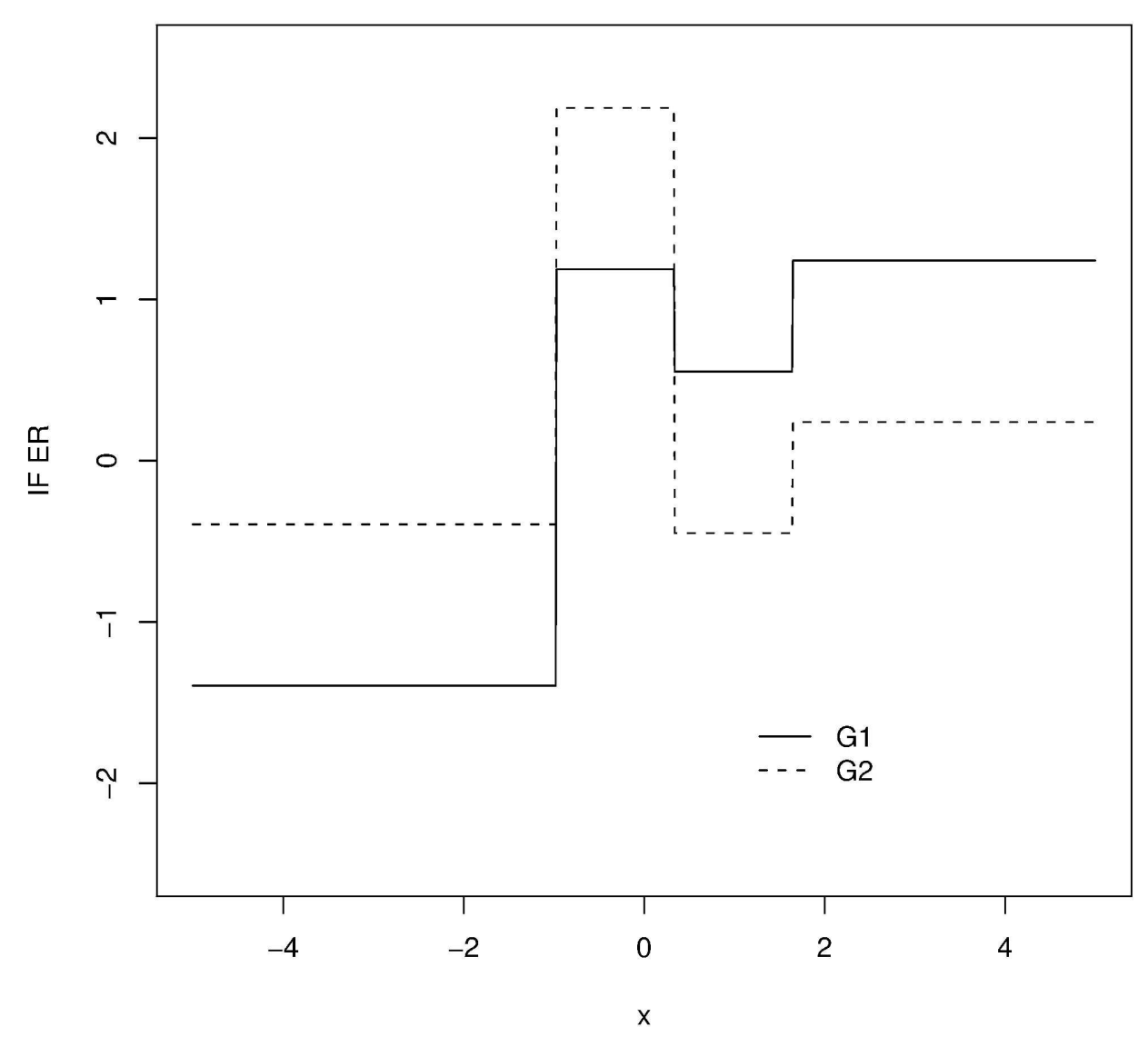

$177 \times 177 m m(600 \times 600$ DPI $)$ 
IF of the training error rate of the 2-means

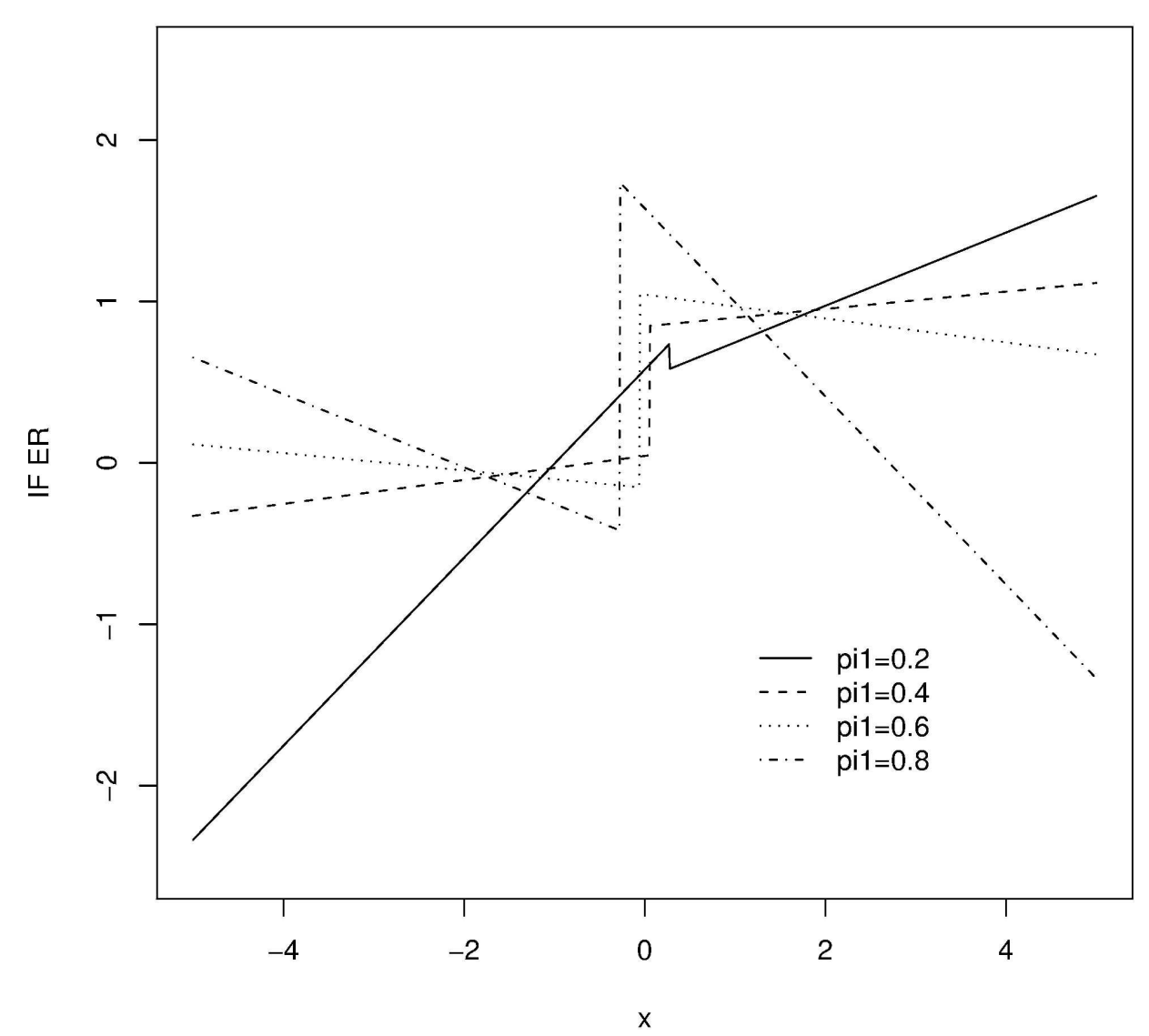

$177 \times 177 \mathrm{~mm}(600 \times 600 \mathrm{DPI})$ 
IF of the training error rate of the 2-medoids

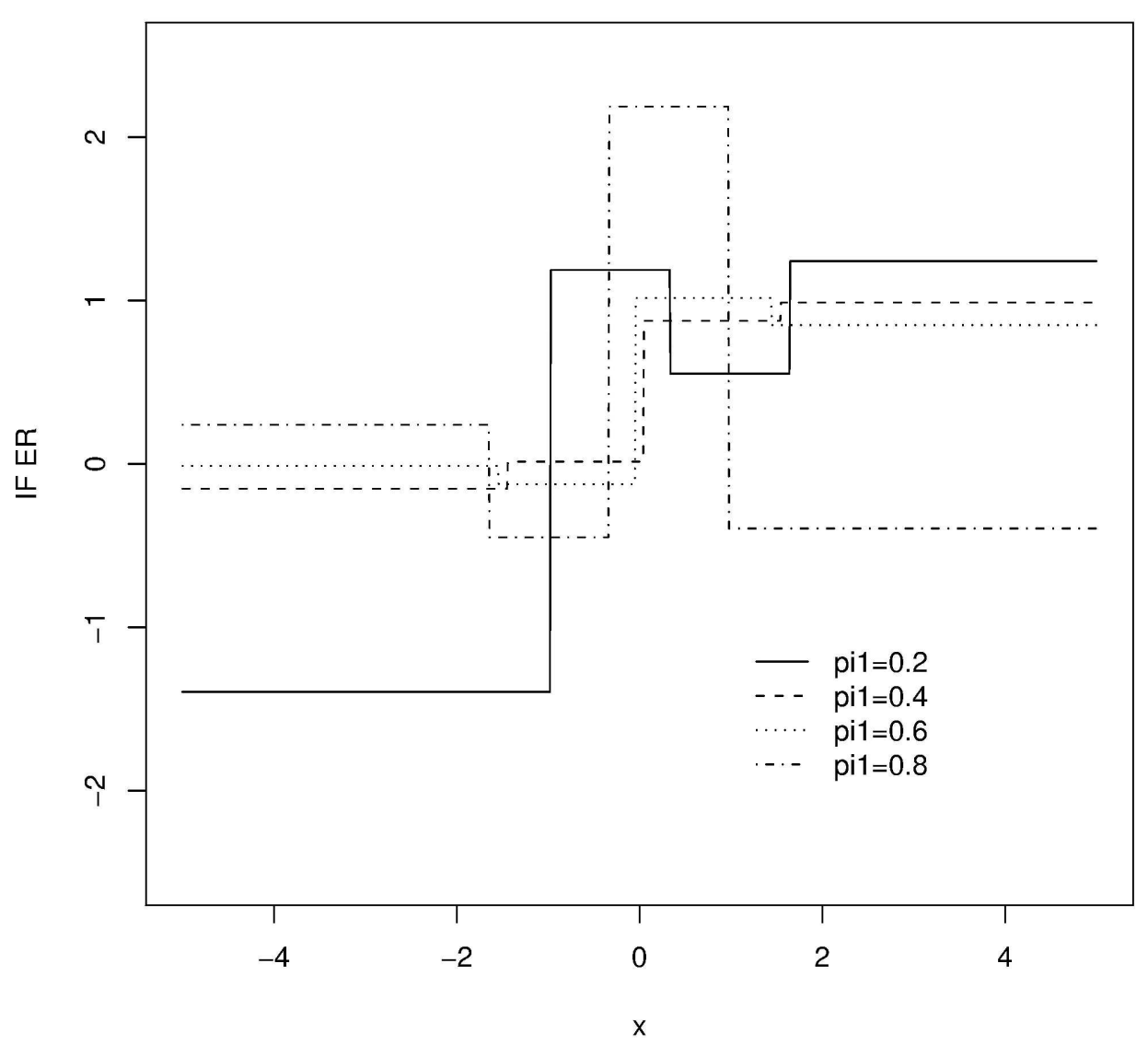

$177 \times 177 \mathrm{~mm}(600 \times 600 \mathrm{DPI})$ 
IF of the training error rate of the 2-means

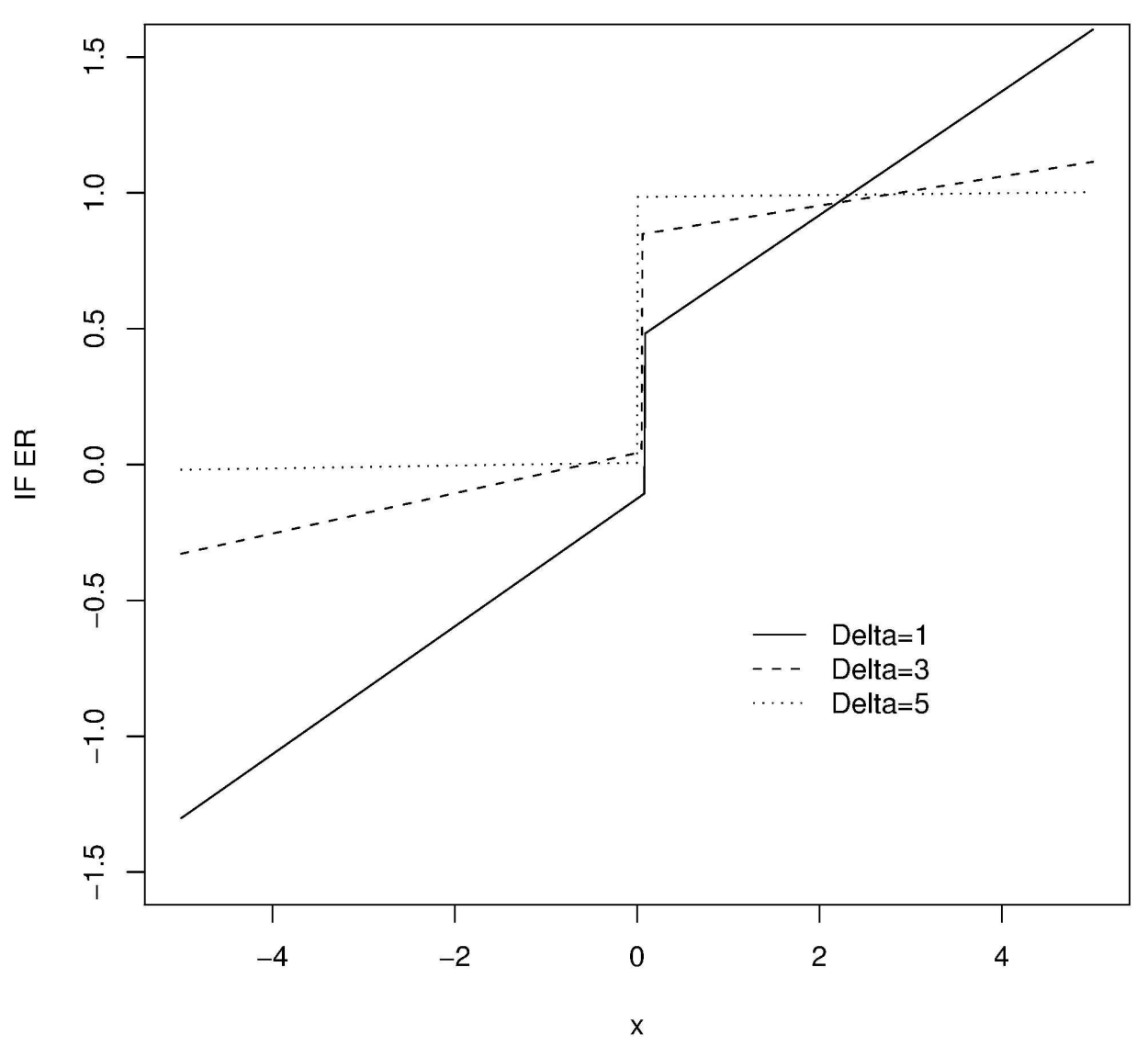

$177 \times 177 m m(600 \times 600$ DPI $)$ 
IF of the training error rate of the 2-medoids

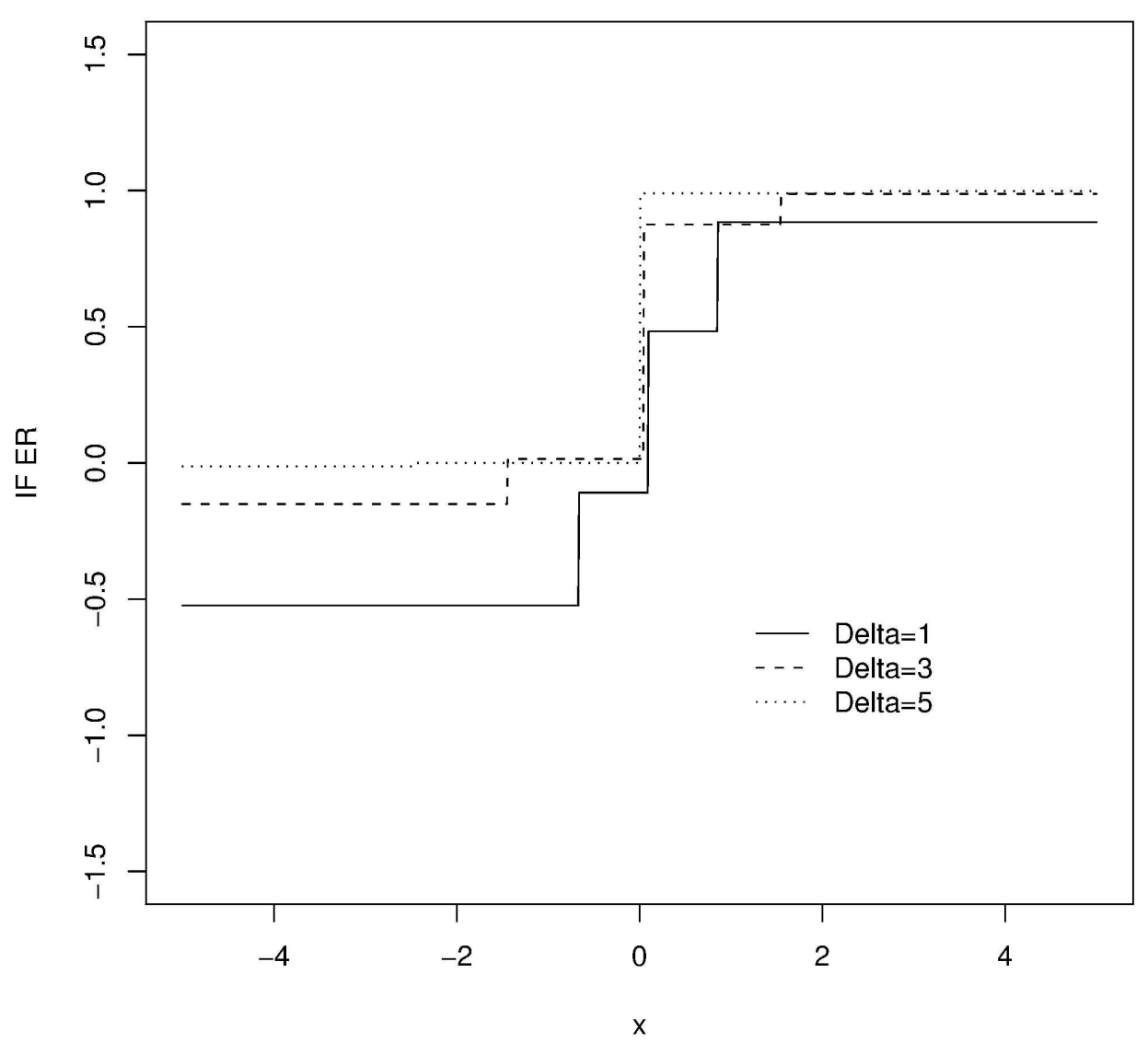

$177 \times 177 \mathrm{~mm}(600 \times 600 \mathrm{DPI})$ 